This article was downloaded by: [80.238.180.123]

On: 21 March 2014, At: 15:36

Publisher: Taylor \& Francis

Informa Ltd Registered in England and Wales Registered Number: 1072954 Registered office: Mortimer House, 37-41 Mortimer Street, London W1T 3J H, UK

\title{
Brain-Computer Interfaces
}

Publication details, including instructions for authors and subscription information:

http:// www. tandfonline.com/loi/tbci20

\section{Workshops of the Fifth International Brain-Computer Interface Meeting: Defining the Future}

Jane E. Huggins ${ }^{a}$, Christoph Guger ${ }^{b}$, Brendan Allison ${ }^{c}$, Charles W. Anderson ${ }^{d}$, Aaron Batista ${ }^{e}$, Anne-Marie Brouwer ${ }^{f}$, Clemens Brunner ${ }^{g}$, Ricardo Chavarriaga ${ }^{\mathrm{h}}$, Melanie Fried-Oken ${ }^{\mathrm{i}}$, Aysegul Gunduz ${ }^{j}$, Disha Guptak ${ }^{k}$, Andrea Kübler ${ }^{\prime}$, Robert Leeb ${ }^{m}$, Fabien Lotte ${ }^{n}$, Lee E. Miller ${ }^{0}$, Gernot Müller-Putz ${ }^{p}$, Tomasz Rutkowski ${ }^{q}$, Michael Tangermann ${ }^{r} \&$ David Edward Thompson ${ }^{s}$

${ }^{a}$ Department of Physical Medicine and Rehabilitation, Department of Biomedical Engineering, University of Michigan, 325 East Eisenhower, Ann Arbor, MI, USA

${ }^{b}$ g.tec medical engineering GmbH/ Guger Technologies OG, Schiedlberg, Austria

c University of California at San Diego, La J olla, CA, USA

${ }^{d}$ Department of Computer Science, Colorado State University, Fort Collins, CO, USA

e Department of Bioengineering, Swanson School of Engineering, University of Pittsburgh, Pittsburgh, PA, USA

${ }^{f}$ The Netherlands Organization for Applied Scientific Research, Soesterberg, the Netherlands

${ }^{g}$ Institute for Knowledge Discovery, Laboratory of Brain-Computer Interfaces, Graz University of Technology, Graz, Austria

${ }^{\mathrm{h}}$ Center for Neuroprosthetics, École Polytechnique Fédérale de Lausanne, EPFL-STI-CNBI, Station 11, Lausanne, Switzerland

${ }^{i}$ Institute on Development \& Disability, Oregon Health \& Science University Portland, OR, USA

${ }^{j}$ Department of Biomedical Engineering, University of Florida, Gainesville, FL, USA

${ }^{k}$ Department of Neurology, Wadsworth Center, NY State Department of Health, Albany Medical College/ Brain Computer Interfacing Lab, Albany, NY, USA

I Institute of Psychology, University of Würzburg, Würzburg, Germany

${ }^{m}$ Center for Neuroprosthetics, École Polytechnique Fédérale de Lausanne, Lausanne, Switzerland

${ }^{\mathrm{n}}$ Inria Bordeaux Sud-Ouest/ LaBRI, 200 Avenue de la Vieille Tour, Talence, France

${ }^{\circ}$ Departments of Physiology, Physical Medicine and Rehabilitation, and Biomedical Engineering, Feinberg School of Medicine, Northwestern University, Chicago, IL, USA

${ }^{\mathrm{p}}$ Institute for Knowledge Discovery, Laboratory of Brain-Computer Interfaces, Graz University of Technology, Graz, Austria

${ }^{q}$ Life Science Center of TARA, University of Tsukuba, Tsukuba, Ibaraki, J apan

'Excellence Cluster BrainLinks-BrainTools, Department of Computer Science, University of Freiburg, Freiburg, Germany

${ }^{\mathrm{s}}$ Department of Biomedical Engineering, University of Michigan, Ann Arbor, MI, United States

Published online: 14 Mar 2014.

To cite this article: J ane E. Huggins, Christoph Guger, Brendan Allison, Charles W. Anderson, Aaron Batista, Anne-Marie Brouwer, Clemens Brunner, Ricardo Chavarriaga, Melanie Fried-Oken, Aysegul Gunduz, Disha Gupta, Andrea Kübler, Robert Leeb, Fabien Lotte, Lee E. Miller, Gernot Müller-Putz, Tomasz Rutkowski, Michael Tangermann \& David Edward Thompson 
To link to this article: http:// dx.doi.org/ 10.1080/2326263X.2013.876724

\section{PLEASE SCROLL DOWN FOR ARTICLE}

Taylor \& Francis makes every effort to ensure the accuracy of all the information (the "Content") contained in the publications on our platform. However, Taylor \& Francis, our agents, and our licensors make no representations or warranties whatsoever as to the accuracy, completeness, or suitability for any purpose of the Content. Any opinions and views expressed in this publication are the opinions and views of the authors, and are not the views of or endorsed by Taylor \& Francis. The accuracy of the Content should not be relied upon and should be independently verified with primary sources of information. Taylor and Francis shall not be liable for any losses, actions, claims, proceedings, demands, costs, expenses, damages, and other liabilities whatsoever or howsoever caused arising directly or indirectly in connection with, in relation to or arising out of the use of the Content.

This article may be used for research, teaching, and private study purposes. Any substantial or systematic reproduction, redistribution, reselling, loan, sub-licensing, systematic supply, or distribution in any form to anyone is expressly forbidden. Terms \& Conditions of access and use can be found at http:// www.tandfonline.com/page/terms-and-conditions 


\title{
Workshops of the Fifth International Brain-Computer Interface Meeting: Defining the Future
}

Jane E. Huggins ${ }^{\mathrm{a} *}$, Christoph Guger ${ }^{\mathrm{b}}$, Brendan Allison $^{\mathrm{c}}$, Charles W. Anderson ${ }^{\mathrm{d}}$, Aaron Batista ${ }^{\mathrm{e}}$, Anne-Marie Brouwer ${ }^{\mathrm{f}}$, Clemens Brunner ${ }^{\mathrm{g}}$, Ricardo Chavarriaga ${ }^{\mathrm{h}}$, Melanie Fried-Oken ${ }^{\mathrm{i}}$, Aysegul Gunduz ${ }^{\mathrm{j}}$, Disha Gupta $^{\mathrm{k}}$, Andrea Kübler, Robert Leeb $^{\mathrm{m}}$, Fabien Lotte ${ }^{\mathrm{n}}$, Lee E. Miller ${ }^{\mathrm{o}}$, Gernot Müller-Putz ${ }^{\mathrm{p}}$, Tomasz Rutkowski ${ }^{\mathrm{q}}$, Michael Tangermann ${ }^{\mathrm{r}}$ and David Edward Thompsons

\begin{abstract}
${ }^{a}$ Department of Physical Medicine and Rehabilitation, Department of Biomedical Engineering, University of Michigan, 325 East Eisenhower, Ann Arbor, MI, USA; ${ }^{b}$ g.tec medical engineering GmbH/Guger Technologies OG, Schiedlberg, Austria; ${ }^{c}$ University of California at San Diego, La Jolla, CA, USA; ${ }^{d}$ Department of Computer Science, Colorado State University, Fort Collins, CO, USA; ${ }^{e}$ Department of Bioengineering, Swanson School of Engineering, University of Pittsburgh, Pittsburgh, PA, USA, ${ }^{f}$ The Netherlands Organization for Applied Scientific Research, Soesterberg, the Netherlands; ${ }^{g}$ Institute for Knowledge Discovery, Laboratory of BrainComputer Interfaces, Graz University of Technology, Graz, Austria; ${ }^{h}$ Center for Neuroprosthetics, École Polytechnique Fédérale de Lausanne, EPFL-STI-CNBI, Station 11, Lausanne, Switzerland; ${ }^{i}$ Institute on Development \& Disability, Oregon Health \& Science University Portland, OR, USA, ${ }^{j}$ Department of Biomedical Engineering, University of Florida, Gainesville, FL, USA; ${ }^{k}$ Department of Neurology, Wadsworth Center, NY State Department of Health, Albany Medical College/Brain Computer Interfacing Lab, Albany, NY, USA; 'Institute of Psychology, University of Würzburg, Würzburg, Germany; ${ }^{m}$ Center for Neuroprosthetics, Ecole Polytechnique Fédérale de Lausanne, Lausanne, Switzerland; ${ }^{n}$ Inria Bordeaux Sud-Ouest/LaBRI, 200 Avenue de la Vieille Tour, Talence, France, ${ }^{\circ}$ Departments of Physiology, Physical Medicine and Rehabilitation, and Biomedical Engineering, Feinberg School of Medicine, Northwestern University, Chicago, IL, USA; ${ }^{p}$ Institute for Knowledge Discovery, Laboratory of Brain-Computer Interfaces, Graz University of Technology, Graz, Austria; ${ }^{q}$ Life Science Center of TARA, University of Tsukuba, Tsukuba, Ibaraki, Japan; ${ }^{r}$ Excellence Cluster BrainLinks-BrainTools, Department of Computer Science, University of Freiburg, Freiburg, Germany; ${ }^{5}$ Department of Biomedical Engineering, University of Michigan, Ann Arbor, MI, United States
\end{abstract}

(Received 12 December 2013; accepted 16 December 2013)

\begin{abstract}
The Fifth International Brain-Computer Interface (BCI) Meeting met on 3-7 June 2013 at the Asilomar Conference Grounds, Pacific Grove, California, USA. The conference included 19 workshops covering topics in brain-computer interface and brain-machine interface research. Topics included translation of BCIs into clinical use, standardization and certification, types of brain activity to use for BCI, recording methods, the effects of plasticity, special interest topics in BCIs applications, and future BCI directions. BCI research is well established and transitioning to practical use to benefit people with physical impairments. At the same time, new applications are being explored, both for people with physical impairments and beyond. Here we provide summaries of each workshop, illustrating the breadth and depth of BCI research and highlighting important issues for future research and development.
\end{abstract}

Keywords: brain-computer interface; brain-machine interface; neuroprosthetics; conference

\section{Introduction}

Brain-computer interfaces (BCI) (also referred to as brain-machine interfaces; $\mathrm{BMI}$ ) provide a direct interface between the brain and technology. Both invasive and non-invasive methods can be used to acquire the signals upon which the BCI is based. The essential element is that the signal source comes directly from the brain, bypassing the normal output pathways in the body. BCIs can be used for communication, prosthetic control, therapy, rehabilitation, and robotics and the list of BCI applications is constantly increasing. Generally, BCIs are described as tools for people with physical impairments, restoring or replacing impaired functions. Here we offer the reader a concise glimpse of relevant topics in the field of BCI research and development.

\section{History and distinctive features of the BCI meeting series}

The International Brain-Computer Interface Meetings occur approximately every three years, bringing together BCI researchers from around the world. The first Meeting, held in 1999, was attended by 50 scientists representing 22 laboratories from the United States, Canada, Great Britain, Germany, Austria, and Italy.[1] The Meetings continued to grow through 2002,[2] 2005, [3] and 2010.[4] The Fifth International BCI Meeting, held on 3-7 June 2013 at the Asilomar Conference Grounds in Pacific Grove, California, USA, was attended by 301 participants from 29 countries representing 165 laboratories. Of these participants, approximately $37 \%$ were students or postdocs. For the first time, the BCI

*Corresponding author. Email: janeh@umich.edu

Authors, after Dr. Huggins as Meeting Chair and Dr. Guger as the workshop organizer, are listed in alphabetical order. 
Meeting included participation by people with physical impairments who could potentially benefit from current BCI technology. The 2013 Meeting was the first to be organized by a Program Committee of BCI researchers from around the world, building on the format established in the first four meetings that were organized by the Wadsworth Center, Albany, New York, USA.

A central fact of BCI research and development is that it is an interdisciplinary endeavor. Success requires contributions from neuroscientists, engineers, psychologists, medical doctors, applied mathematicians, computer scientists, clinical rehabilitation specialists, and more. Expertise in all these disciplines, as well as interactions with the intended users, is necessary for productive research and for the development and testing of useful applications. Communication among disciplines and the collaborations that such interactions foster are essential for continued progress. Although many meetings now offer BCI sessions, the BCI Meeting series is unique in providing an appropriately comprehensive and international venue that brings together all relevant disciplines and increasingly includes participation from potential users of BCI technology.

Other distinctive characteristics of the BCI Meetings are the large student participation and the retreat format, in which on-site housing and included meals promote interactions between diverse attendees. With a theme of 'Defining the Future', this Fifth BCI Meeting expanded on the interactive workshops that are distinctive of the BCI Meetings by accepting formal workshop proposals from the BCI community.

\section{Organization of workshop summaries}

The final full day of the Meeting was devoted to workshops. This report is composed of summaries of the individual workshops, grouped by topic to develop the different themes present. The organizers are listed for each workshop and all additional presenters are identified. While it is impossible to recreate the interactive experience of the workshops themselves, these summaries provide an introduction to their important elements, identify resources for further exploration, and present the resultant conclusions or future directions.

The translation of BCI to practical use for people with physical impairments has been of great interest during many of the Meetings in the BCI Meeting Series. In the 2013 Meeting, two clusters of workshops advanced this goal. One set discussed practical issues for independent $\mathrm{BCI}$ use and $\mathrm{BCI}$ experiments in a home environment. A second set of workshops focused on defining the best practices, conventions, and standards necessary to a maturing research area.

Signal selection and interventions intended to modify brain signals formed the basis of several workshops, with discussions of the types of signals that can be used and the potential for optimizing performance by harnessing brain plasticity. Another set of workshops brought together groups interested in established BCI areas with special application characteristics. Finally, about a third of the workshops explored future developments in BCI research, some building on long histories of BCI research leading to emergent breakthroughs, with others identifing new areas into which BCIs could expand.

\section{Translational focus}

\section{Conducting BCI experiments in the home}

Organizer: Chuck Anderson

Presenters: Patti Davies, William Gavin, and Marla Roll

Expanding BCI research from the laboratory into subjects' homes can pose a challenge for labs whose personnel lack experience conducting home-based research and further complications arise when working with clients with severe motor impairments. Successful transition to home studies requires defining how to (1) recruit, inform, and obtain consent from clients in their homes, (2) minimize interference from electrical sources and activity in the clients' environment, (3) design protocols and applications that are useful and functional in the client's home and (4) collect BCI users' experiences and impressions of EEG equipment and BCI protocols.

Recruitment of subjects is facilitated through collaboration with local academic, supportive, and professional organizations in rehabilitation, amyotrophic lateral sclerosis, multiple sclerosis, cerebral palsy, and spinal cord injury. Obtaining appropriate approval from an ethics or institutional review board requires defining the subject population to recruit from, the BCI protocols to be used, the means of obtaining informed consent from subjects and caregivers, and the steps to be taken to maintain the privacy of the subjects. Preliminary telephone or personal discussions with caregivers are very helpful.

Electrical interference and distractions are often difficult to remove in the home environment. Some sources of electrical noise are difficult to identify. Hospital beds, even when switched off, will still generate interference and must be unplugged from the outlet. Switching off computers, screens, and lights in the area can also help. While removal of noise sources can be a temporary measure to facilitate an experiment, ultimately, the BCI must exist in an environment containing the medical equipment necessary for the long-term health of a user with severe impairments. Real-time filtering methods can be helpful, such as notch filters to remove power-line frequencies and band-pass filters to remove low frequencies due to eye blinks and high frequencies due to muscle twitches. Subjects can also be distracted by over-eager coaching from caregivers, and movement and noise from bystanders and pets. 
Most researchers report using BCI2000 or custom software for in-home experiments. P300 speller applications are the most common, as well as motor imagery applications. Standard protocols that are usable in the lab with unimpaired subjects may not be useful in the home. P300 waves may be harder to detect in the home due to interference issues. Subject concentration may drift due to distractions. Careful attention to the presentation of visual stimuli may be needed, requiring appropriate lighting conditions and appropriate contrast and colors on the computer screen. Researchers should bring an adjustable bed stand for holding the computer screen and positioning it for optimal viewing by the subject. Advance information on the physical space limitations can ensure ample space for safely placing recording equipment near the subject.

The collection of a subject's experience is essential. After each session, ask the subject and the caregiver for their impressions of which protocols and EEG systems they prefer. Ask which BCI applications they would most like to see.

In summary, workshop participants agreed that the discussions of possible problems and ways of addressing the problems when recording in the home were very valuable. A central repository for all to share lessons learned would be welcome.

\section{Independent home use of BCI: requirements for translation and evaluation}

Organizer: Andrea Kübler

Presenters: Theresa Vaughan, Eric Sellers, and Elisa Holz

While research on BCIs for communication and control has increased almost exponentially over the past 20 years, BCIs remain a last-resort mode of communication due to functional limitations, modest accuracy, and low speed in comparison to other augmentative and alternative communication solutions. Nonetheless, BCIs may be the only viable option of restoring independent communication and autonomy for some people who are severely disabled. Considerable effort has been invested in improving signal detection, speed, accuracy, and reliability.[5-11] However, studies in target populations are far less common.[12] There remains a translational and reliability gap that must be filled by studies of BCI use by target populations and long-term in-home studies to improve BCI reliability.[13,14]

$\mathrm{BCIs}$ are fast becoming effective communication and control devices in the laboratory. However, they must be shown to work in real life and to provide capabilities that improve people's lives. They must be simple to operate, need minimal expert oversight, be usable by people who are extremely disabled, and provide reliable, long-term performance in complex environments. Their capacity to satisfy these demanding criteria can only be determined through long-term studies of independent home use by target user populations. Translational research to establish BCIs' clinical value must answer four questions. (1) Can the BCI be implemented in a form suitable for long-term home use? (2) Who needs and can use the BCI? (3) Can the home environment support BCI usage and is it actually used? (4) Does the BCI improve the user's life [14-16]?

The user-centered approach provides a standardized framework to design and evaluate translational and reliability studies and enables comparison between BCI-based applications for communication and control. This iterative process of development and feedback between researchers and users can lead to increased product refinement. User-centered design defines usability as effectiveness, efficiency, and satisfaction. For BCI-controlled applications, effectiveness is equivalent to selection accuracy and efficacy to the information transferred per time unit and the effort invested (workload). Satisfaction with a device can be assessed for general and $\mathrm{BCI}$-specific aspects and includes the match between user and technology.[13,17-22]

Studies in the user's natural environment are needed to prove the usability and feasibility of BCI devices for daily use and to identify challenges and additional applications. The BCI application Brain Painting was installed in the home of a person locked-in by ALS and the usability of the BCI device was continuously evaluated by the end-user. This person has used the BCI for more than a year with only the help of her family. BCIcontrolled Brain Painting not only improves quality of life, but also enables social inclusion.[21,23-25]

Further research is needed on usability, system robustness and convenience, training and technical support, subject inclusion criteria, recruitment, consent, and retention. The workshop members discussed issues, obstacles, and solutions from which a decision algorithm for bringing BCIs to end-users was derived.[26]

\section{Augmentative and alternative communication for BCI 101}

Organizer: Melanie Fried-Oken

Presenters: Greg Bieker, Jane E. Huggins, Aimee Mooney, and Betts Peters

The field of Augmentative and Alternative Communication (AAC) provides a framework for device design, clinical assessment, and intervention for people with complex communication needs.[27] AAC is more than just devices; it is a human process of information transfer.[28] AAC includes both unaided approaches, such as eye blinks for yes/no, and aided approaches, such as communication boards or speech-generating devices. BCI-based communication systems, like other aided AAC approaches, should be customized to each user's needs 
and abilities. Before adopting the BCI system, the user should consider: (1) the language of the device, which may include letters, icons, photos or phonemes, and the contribution of language models for word prediction or completion; (2) the output of the device, including speech output and/or visual displays (i.e., text on a computer screen); (3) the input mechanism or how one selects the language units in the device (e.g., event-related potentials, steady-state visual evoked potentials, or multimodal access incorporating eye gaze, head mouse or single switch control [29]); and (4) the user's own preferences and feedback. The intersection between BCI and AAC research and development is significant, especially as we consider BCI systems as assistive technologies for people with severe speech and physical impairments.[30]

AAC-BCI collaborations should include hybrid BCI systems that incorporate access methods such as eyegaze; systems with GPS or speech-recognition that determine the context of a conversation and provide appropriate vocabulary; algorithms that identify error-related potentials for error correction; and automatic system adaptations that infer and adjust to changes in the user condition (e.g., fatigue, emotional state, cognitive workload). Technical challenges such as synchronizing $\mathrm{BCI}$ system timing with $\mathrm{AAC}$ components and adequate $\mathrm{BCI}$ accuracy must be addressed since each type of BCI will provide a different input interface for AAC. Successful development of BCI input for AAC devices would benefit from prioritizing the $\mathrm{BCI}$ signal sources to support AAC devices and establishing standards and research guidelines for $\mathrm{BCI}$ inputs to $\mathrm{AAC}$ devices.

As BCIs transition to clinical use, a feature-matching procedure will help determine whether BCI as an access method is appropriate for each user. The Matching Person to Technology Model [31] provides guidelines for implementing this process. BCI systems must be adaptable to the context for communication, the purposes of communication,[32] and the role of communication partners or caregivers for message generation.

Finally, the driving principle for BCI research and development must be the user. As a Participatory Action Research challenge,[33] the design and implementation plans must be shaped by feedback from people with severe speech and physical impairments and their families and caregivers.[34] This was emphasized by a presentation from Greg Bieker, the only person with locked-in syndrome present at the BCI meeting. [35,36]

\section{Standardization \\ BCI software platforms and standards}

Organizer: Clemens Brunner

Presenters: Gerwin Schalk, Jürgen Mellinger, Christian Kothe, Robert Oostenveld, Armin Schnürer, Jussi Lindgren, and Febo Cincotti
This workshop utilized a discussion format to build consensus among participants with a goal of increasing interoperability and compatibility between BCI labs worldwide. Participants discussed current software tools and potential synergies to improve their interoperability. Discussion goals were (1) to identify currently available software solutions for BCI platforms; (2) to become aware of issues and possible solutions when combining components from different platforms; (3) to discuss a suitable common data format for BCI research; and (4) to identify future steps to address issues discussed in this workshop.

The most commonly used BCI software platforms [37] are: BCI2000,[38] OpenViBE,[39] BCILAB with lab streaming layer (LSL),[40] FieldTrip,[41] a proprietary solution by g.tec, and the TOBI platform.[42] A major topic for many participants was to ensure proper support for hardware devices, which was important for software developers, end-users, and hardware companies. We concluded that to avoid reinventing the wheel, we should reuse existing signal acquisition modules in other platforms. The feasibility of this approach has been demonstrated by integrating the OpenViBE acquisition server with BCILAB's LSL, enabling the extended OpenViBE module to stream data in the LSL format.

Software developers also stated that support for hardware with completely open specifications was much easier to implement, which means that data transmission protocols should be documented and not kept secret. However, not all companies agreed, since proprietary protocols enable them to restrict the usage of their devices to tested and supported software environments. In this workshop, we had companies representing both open and proprietary strategies.

Secondly, we discussed ideas for a common file format in the BCI community. People seemed to be very interested in establishing a unified file format, judging by discussions with several leading developers and activity on the workshop mailing list. Creating one common data format would dramatically improve interoperability, and a candidate format later proposed in the mailing list was XDF (extensible data format, code.google.com/p/xdf/).

Finally, the next steps towards BCI software standards will be to continue the discussion about a common file format on the dedicated workshop mailing list (which is now open to the public at groups.google.com/d/forum/bci-standards). Furthermore, a common protocol to exchange data between different platforms could be established by using the TOBI and/or LSL standard interfaces; it remains to be seen if the major platforms adopt one or both protocols (at least in addition to their native communication protocols). 


\section{BCI performance metrics}

Organizer: David Edward Thompson

Presenters: Shangkai Gao, Lucia Quitadamo, Luca Mainardi, and Khalil Laghardi

The workshop on BCI performance assessment included presentations of existing metrics such as the Information Transfer Rate (ITR),[43] Extended Confusion Matrices (ECM),[44] BCI-Utility,[45] and user-experience-based metrics,[46] as well as discussions of performance measurement in general. BCIs rarely meet the underlying assumptions for ITR, such as equal probability of classes.[47] The ECM metric has the advantage of correct handling of abstentions (selections that do not produce an output).[44,48] The BCI-Utility metric measures expected benefit over time and can be adapted to capture the effects of different enhancement methods such as automatic correction.[45] The uFEEL framework facilitates assessing the overall user experience of a BCI, and includes different types of metrics, such as NASA's Task Load Index.[46]

The second half of the workshop included a group discussion on metric choice, and presentations with further discussion on contentious practices in performance reporting. Customizable parameters (aka free parameters) in metrics lead to inconsistent usages and prevent valid comparisons even between studies reporting the same metric. Such dangers can be overcome if BCI researchers standardize the values of these parameters, creating field conventions and therefore consistent metric calculation. Performance is sometimes also artificially inflated through task choice or non-standard calculation of variables in the equations for each metric. The workshop concluded with a group discussion and decision to collaboratively author a journal paper with the results of the workshop, extended by further discussion.

That paper is currently in submission to the Journal of Neural Engineering special issue on the BCI Meeting, under the title of 'Performance Measurement for BrainComputer or Brain-Machine Interfaces: A Tutorial'. The paper contains checklists for methods sections for both discrete and continuous BCIs, and summaries and specifics on various metrics discussed during the workshop.

\section{BCI certifications, guidelines and other standards}

Organizer: Brendan Allison

Presenters: Jane E. Huggins, Shangkai Gao, Anton Nijholt, Tobias Kaufmann, and Armin Schnuerer

As BCI research gains momentum, there is a growing need for standards including ethical guidelines, terms and definitions, and reporting guidelines to form the infrastructure of a BCI community. These include methods to calculate information transfer rate (ITR) and adequately describe participants' selection and rejection criteria and other characteristics such as the nature of any disabilities. Indeed, surveys conducted at the 2010 International BCI Meeting [49] and the 2013 International BCI Meeting showed recognition of a pressing need for such standards. Additionally, many groups utilize different reporting techniques that could lead to confusion and undue inflation of reported $\mathrm{BCI}$ performance.[47,50] The launching of the BCI Journal and the on-going efforts to establish a BCI Society may provide the formal entities to develop and entrench such standards.

A particular concern was appropriate training and qualification for researchers providing BCIs to users, particularly users with physical impairments. For example, researchers might be expected to demonstrate familiarity with the challenges of obtaining informed consent from a user who is not able to speak. Such guidelines for ethical research are naturally the responsibility of an ethics or Institutional Review Board (IRB). However, some BCI work may be conducted through entities that do not seek IRB approval (such as industrial research), or through institutes whose IRBs may be unfamiliar with the unique issues associated with BCI research for people with limited communication. The emerging BCI Society could formalize such expectations, later leading to a test or formal certification, but this may be premature and could impede BCI research. Establishment of a formal certification credential is a large undertaking (as illustrated by the assistive technology provider credential from RESNA - Rehabilitation Engineering and assistive technology Society of North America, www.resna.org). However, organization guidelines for ethical behavior are a common feature of professional societies.

Consistent reporting guidelines are another area of concern. Many articles do not publish critical information about how subjects were selected or rejected, details of subjects' medical background and capabilities, and how ITR was calculated. Groups often select subjects with BCI experience who are expected to yield promising results and ignore the difficulties of generalizing these results to the general population. Further, groups often use inappropriate methods to report ITR, such as failing to account for the time between selections. Adequate reporting of results requires a clear description of the method of calculating ITR, including ways in which the ITR calculation may not reflect real-world performance. A BCI Society could also manage media reporting guidelines. Many BCI articles in the popular media are inaccurate or misleading.[51] A BCI Society could provide a respected centralized entity to respond to bad reporting and publicize established guidelines such as the Ingelfinger rule, which cautions against publicly announcing work that has not passed peer-review. This society would presumably have a central website that 
could include standards and guidelines to help researchers, especially people new to BCI research.

These issues of ethical and reporting guidelines are increasingly crucial as BCI research becomes a mature, mainstream research field. Standardization involves a variety of different elements, many of which will require further discussion and debate to reach consensus within the BCI community.

\section{The brain side of the $\mathrm{BCI}$}

\section{Neuronal ensembles for BCI: local field potentials and electrocorticography}

Organizer: Aysegul Gunduz

Presenters: Dora Hermes, Christoph Kapeller, Dan Moran, Bijan Pesaran, Nick Ramsey, Gerwin Schalk, and Wei Wang.

Recordings from neuronal ensembles, namely local field potentials (LFPs) and electrocorticography (ECoG), have attracted increasing interest in the field of BCIs due to the stability and specificity of these recordings for the interpretation of behavioral and cognitive tasks.[52] LFPs and ECoG represent cumulative afferent synaptic activity, which exhibits broad spectral ranges that modulate with behavior. High gamma activity ( $\sim 70$ to $\sim 300 \mathrm{~Hz}$ ), unique to intracranial field potentials, is a prime candidate for $\mathrm{BCI}$ control due to its high correlation with behavior and fine localization.[53] A recent ECoG study demonstrated three-dimensional control of a robotic limb in a subject with tetraplegia through high gamma modulation ([54], also see [55,56]). Still, invasive approaches can only be clinically viable when the expected benefits outperform non-invasive solutions and outweigh the potential risks. Improving system safety, durability, and performance is not only of utmost importance for the technical viability of invasive BCIs, but is also of great ethical concern.

Although there are long-term studies demonstrating the stability of these signals in animals,[57] present data on the long-term safety of invasive implants in humans are limited.[58-60] Recently, the Neuropace Responsive NeuroStimulator ${ }^{\circledR}$ (RNS), a chronic subdural recording and stimulation system,[61] gained recommendation for FDA approval from the advisory panel, which concluded that its clinical benefits outweigh the risks of its use,[62] paving the path for future cortically implanted systems. Other studies are under way to investigate the signal fidelity of recordings from epidural electrodes, whose position over intact dura results in a lower risk of infection to the brain. Studies in humans show reduction in signal amplitude over the dura compared to subdural recordings.[63,64] Still, animal studies demonstrate the viability of epidural BCI control. $[65,66]$

Apart from stability and safety, questions remain regarding the optimal design and implantation of recording electrodes. LFPs are often simultaneously recorded with single unit activity using single-contact multi-electrode arrays, although a recent study shows that the optimal laminar depths for these two modalities are different due to the nature of the synaptic potentials versus action potentials.[67] Optimal subdural electrode diameter and density for BCI use in humans remains mostly unexplored. Denser electrode designs provide more detailed information,[68-70] but in turn will increase processing and telemetry requirements in fully implantable systems. Animal studies will remain important to uncover optimal ECoG electrode grid designs and guide human studies.[71]

\section{Cognitive processes and brain-machine interfaces}

Organizer: Ricardo Chavarriaga

Presenters: Scott Roset and Nathan Evans

A great deal of BMI research focuses on using neural correlates of motor activity to operate devices. However, neural correlates of cognitive processes can also be used to enhance human-machine interaction. Increasing evidence supports the decoding of neural activity related to attentional mechanisms $[72,73]$ as well as sensory processing [74] and mental workload.[75,76]

The idea of decoding neural correlates of these processes began over 60 years ago with Grey Walter's pre-cognitive carousel [77] and was advanced by the DARPA Biocybernetics program (1974-1978) on enhancing man-machine systems. This work supported early ERPs studies as a measure of workload [76] that showed how the P300 amplitude in secondary tasks is modulated by the difficulty of the primary task.[78] The P300-based BCI [79] also sprang from this line of research. Beyond the P300 BCI, however, cognitive processes are not widely exploited by current BCIs. A potential drawback is the need for secondary tasks. However, tapping into processes that are naturally elicited during interactions may be a more transparent and intuitive way to enhance current BMIs (see also the workshop Passive BCI - using neurophysiological signals that reflect cognitive or affective state). Examples of these processes include the prediction of movement intention and error-related neural activity.

Neural activity preceding actions [80] is currently explored to predict onset of self-paced movements [81,82] and interpreting motor control and volition.[83] For example, it can improve motor neuroprostheses by providing a tighter coupling of the intention-related brain activity and movement execution with a prosthesis or robotic device. This may promote beneficial plasticity after brain injuries such as stroke.[84] These correlates can also be exploited in applications for able-bodied users such as a car-driving scenario that decoded selfpaced decisions on braking and steering.[85] 
Error-related neural correlates resulting from assessing the correctness of actions have been identified with several recording techniques [86-88] and across different tasks [89] and feedback modalities.[90] Interestingly, these signals can be decoded on a single-trial basis and used to correct erroneous decisions.[91,92] Alternatively, they can be used to improve the BMI using the reinforcement-learning paradigm. $[86,87,93,94]$ Despite these advances, it has yet to be confirmed whether these correlates can be exploited in a more continuous manner, e.g., detecting errors not strongly synchronized to external stimuli as well as decoding information about the magnitude of such errors.

Another challenge is to fully validate the feasibility of decoding cognitive processes during complex tasks and real scenarios of human-machine interaction. This may require hybrid approaches simultaneously monitoring different brain processes and exploiting multimodal recordings.[95,96] A potential avenue is to extend current methods to capture the neural dynamics linked to these processes, e.g., by extracting features based on functional connectivity patterns.[97,98]

Last but not least, BMIs can be a tool to understand the neurophysiology of cognitive processes, enabling study of these processes in interactive environments instead of standard constrained paradigms. A recent example shows how BMI paradigms can be used to study subjective senses of limb ownership and agency, [99] key factors to achieve intuitive, efficient control of motor neuroprosthetics.

\section{Is plasticity necessary for good BCI control?}

Organizer: Aaron Batista

Presenters: Dan Moran, Patrick Sadtler, Karunesh Ganguly, Eric Pohlmeyer, Amy Orsborn, Steve Chase, and Andy Jackson

Efforts to improve BCI performance must answer the design decision about whether to focus on developing the most effective decoding algorithms, or whether to rely on neural plasticity to allow users to improve device performance through experience. Perhaps a hybrid approach exists, wherein decoding algorithms can be designed that harness neural plasticity.

A special aspect of this workshop is that nearly all speakers employ invasive approaches in the development of BCIs. Each of the studies provided an impressive example of the quality and speed of control that invasive BCI approaches can provide. Decoder adaptation and neural plasticity can combine to yield performance improvements and robustness to interference, and boost long-term retention of performance. Offline consolidation overnight can improve BCI learning. Reinforcement learning can be used to shape BCI performance without detailed error feedback. The envelope of performance can be pushed using intracortical techniques, enabling monkeys to control two BCI cursors simultaneously. This work highlighted an important lesson for the field, that top performance can only be achieved with highquality neural recordings.

However, there are specific and intriguing limitations on the extent to which neural plasticity can boost BCI performance. The neural strategies that animals use to learn to control a BCI involve a search for an optimal solution among the natural constraints that exist within the neural space. Additionally, distorting the relationship between vision and action allows direct study of BCI learning, and shows that visuomotor rotations are learned as readily under BCI control as they are for actual arm movements. However, gain changes cannot be learned readily in a BCI context, while they are readily learned for real arm movements. This highlights a shortcoming in current decoder designs - decoder algorithm performance might be fundamentally limited since the understanding of how motor cortex controls natural arm movements is incomplete

As a whole, this workshop shows how investigators are pushing the BCI learning paradigm to the very limits of performance, and understanding how the brain reorganizes to achieve BCI control. An overarching message of the workshop is that even as we attempt to develop a range of therapeutic options to suit a diverse patient population, we must maintain an emphasis on how (and by how much) the brain can reorganize to make BCI performance better, more robust, and more long-lasting.

\section{Teaching the BCI skill: feedback and human training approaches}

Organizers: Fabien Lotte, Reinhold Scherer, and Anatole Lécuyer

Presenters: Jozef Legény, Elisabeth Friedrich, Moritz Grosse-Wentrup, Jonathan Wolpaw, Chadwick Boulay, Deniz Erdogmus, Avary Kent, Sergio Varona-Moya, and Jeremiah Wander

Although EEG-based BCIs are promising for numerous applications, e.g., rehabilitation or gaming, due to low reliability they mostly remain laboratory prototypes.[100] Poor BCI performance is partly due to imperfect EEG signal processing algorithms but also to the user, who may not produce reliable EEG patterns. Indeed, BCI use is a skill requiring proper user training.[100] If a user cannot perform the desired mental commands, no signal processing algorithm can identify them. Nevertheless, many research efforts focus on signal processing, largely ignoring the human in the loop.

The most realistic and most difficult approach to BCI design is to optimize the co-adaptation between the user and the BCI.[100] This adaptation should engage, reward, and guide brain plasticity.[100] One approach to the still open question of optimal user training and co-adaptation 
is to use adaptive training protocols that first explore various mental tasks (e.g., motor imagery or mental rotation) and then train the user to control the most efficient ones.[101] In general, adapting the training protocol to each user is beneficial: individual channels, frequency bands and mental task selection, individual classifier updates, and co-adaptive learning (with online classifier adaptation) all proved useful for training BCI users.[101103]

To master a BCI, the user must understand how the BCI works and how to use it. One method of providing this understanding is well-designed feedback that is made clear and easy to perceive by integration with the environment, collocating the feedback with the BCI stimulations (e.g., flickering items for SSVEP).[104] Multimodal feedback has also been explored, e.g., by combining visual feedback with haptic feedback [104] or audio feedback,[105] the latter being particularly useful for patients with low vision.

Effective learning also requires motivation, which can be accomplished with engaging training environments such as virtual reality,[105] or immersive virtual reality.[104] Games [106] also positively impact the user experience and/or learning efficiency. Attention seems to be another essential factor for successful BCI control. Indeed, it has been shown that fronto-parietal gamma power could predict users' performance with a sensorimotor rhythm (SMR) BCI and seems associated with attentional networks.[107] Training BCI users to increase their attentional levels using fronto-parietal neurofeedback is therefore a possible approach to improve $\mathrm{BCI}$ performance.

Research on BCI learning can benefit from existing fields of study on learning. Learning to use an SMR-BCI led to similar cortical changes as a motor learning task.[108] This suggests that motor learning theory could inform improved BCI training protocols. Literature on instructional design and educational psychology contains guidelines to ensure efficient skill learning which are rarely satisfied by BCI training protocols. Therefore such guidelines may also improve the efficiency of $\mathrm{BCI}$ training.[109]

Overall, feedback and user training approaches are a valuable approach to boost the currently modest BCI performance. This workshop also showed the continuing lack of knowledge about user learning in BCI (e.g., for patient learning, stable skill acquisition or feedback content), thus providing exciting opportunities and challenges for future research.

\section{BCI special interest applications}

Non-invasive BCI control of grasp neuroprosthesis in high spinal cord injured humans

Organizers: Gernot Müller-Putz and Rüdiger Rupp

\section{Presenter: Martin Rohm}

The bilateral loss of the grasp function associated with a complete or nearly complete lesion of the cervical spinal cord severely limits the affected individuals' ability to live independently and retain gainful employment post-injury. Thus, it represents a tremendous reduction in the patients' quality of life. Any improvement of a lost or limited function is highly desirable, not only from the patient's point of view but also for economic reasons. If tendon transfers are not applicable due to the unavailability of muscles under voluntary control, neuroprostheses based on functional electrical stimulation (FES) provide a (non-invasive) option for functional improvement of the upper extremity function.[110] In particular, hybrid-FES systems consisting of FES and active orthotic components seem to be effective in restoration of a relevant grasp function.[111]

On the other hand, EEG-based BCIs may be a valuable component in a neuroprosthetic user interface. A major advantage over other assistive devices is that a $\mathrm{BCI}$ can be operated independently of residual motor functions. Further, motor-imagery (MI)-based BCIs have enormous potential to provide natural control of grasping and reaching neuroprostheses using signals recorded from brain areas associated with upper extremity movements, especially for individuals with a high spinal cord injury (SCI). The combination of traditional assistive devices with a BCI and the use of combinations of brain activity and movement signals for their operation (hybrid-BCI) open new possibilities for real-time control of a neuroprosthesis autonomously by the end-user with an SCI.

We reviewed the state of the art in non-invasive grasp neuroprosthesis as well as in hybrid brain-computer interfacing.[42,112] Subsequently, we presented the actual state of the art in non-invasive BCI-controlled neuroprosthesis [113-116] with an emphasis on the real application of these systems in individuals with high spinal cord injury.[117,118] The highlight of the workshop was the online demonstration (via teleconference) of an end-user with SCI at Graz University of Technology operating the applied neuroprosthesis with a non-invasive hybrid BCI operated by EEG for switching between two different grasp types (palmar and lateral grasp) and a shoulder position sensor for the finger position. During this demonstration we could observe the functionality of the system. After the demo, workshop participants used the opportunity to ask questions of the user.

Future activities in this research area are the adaptation of (hybrid) BCI systems to the needs of the users as well as identification of new control signals from the EEG. Most prominent here is the direct decoding of complex arm or hand movements from the non-invasive 
EEG, allowing a pure EEG-based control of the neuroprosthesis.

Overall, the workshop demonstrated that the combination of EEG-based BCI and a non-invasive/invasive neuroprosthesis works. An EEG-based BCI can provide hand movement control for individuals with SCI. Further, it provided the first examples of the use of EEG-based (hybrid) BCIs for the control of hand and elbow function in individuals with SCI.

\section{Current state and future challenges in auditory BCI}

Organizers: Michael Tangermann, Jeremy Hill, and Martijn Schreuder

Auditory BCI experimental approaches are many and varied,[119-124] yet they can be characterized by the categories (a) application mode (online/offline), (b) EEG features exploited, (c) application type, (d) field of activity, (e) user interaction (none, direct or implicit feedback), (f) software basis and interfaces, and (g) application software basis.

After an overview of the young research field, workshop participants completed forms (with multiple answers allowed). The results from 22 participants showed that the majority of labs applied their auditory BCI system online (14 participants) with ERP features slightly preferred over oscillatory features (13). All applications targeted communication (22), mostly within the field of BCI basic research (16), while industrial applications (1) and clinical applications (2) played a minor role. Only 13 of these systems made use of direct feedback to users, and BCI2000 (11) was the preferred software platform for the BCI core (custom-programed was second with 6). Applications were mostly developed in Matlab (14), followed by $\mathrm{C}^{++}$(9) and Python (1). When asked to identify bottlenecks to auditory BCIs, participants mentioned classification performance (8) before funding (5) and effectiveness of auditory paradigms (4). Interestingly, reliability of the BCI system (3) and software timing (2) played a minor role only, indicating the quality of the BCI toolboxes. As in the general BCI field, auditory BCIs are not transferred to clinical applications and patient use on a regular basis, although patient applications were acknowledged as a priority for auditory BCI research.

Auditory BCI is a small but rapidly growing field that is quickly forming a novel growing community. Workshop attendees created an email list (https:// lists.tu-berlin.de/mailman/listinfo/neuro-auditorybci) to strengthen connections between active auditory BCI research labs, by sharing and discussing research questions, distributing conference dates, posting job announcements, etc.

\section{BCIs in stroke rehabilitation}

Organizer: Christoph Guger

Presenters: Donatella Mattia, Junichi Ushiba, Cuntai Guan, Surjo R. Soekadar, and Woosang Cho

Lately, BCI systems have become increasingly useful in the context of stroke rehabilitation. The majority of those BCI systems are based on motor imagery activating the sensorimotor cortex. This activity is translated into continuous control signals for rehabilitation devices. While these devices can be virtual reality set-ups that allow the users to see moving limbs of avatars, studies in patient populations have also successfully used functional electrical stimulation, robotic devices, or exoskeletons attached to the patients' paralyzed limbs. Clinical studies have been completed in a variety of settings [125-127] and the physiological effects of BCI stroke rehabilitation have been studied.[127,128]

A variety of BCI hardware and software components to support this research are available.[129-139] These systems analyze brain activity from the sensorimotor region, extract relevant information, and perform a classification in order to control rehabilitation devices in real time. The variety of BCI systems for stroke rehabilitation was discussed, revealing the breadth of relevant components and remaining questions for optimal design. For example, such systems can use robotics that move parts of the body or the whole body, or they can use functional electrical stimulation or apply tactile or visual stimulation to improve rehabilitation outcomes. As yet unresolved are the questions of which feedback modality and BCI training approach best improves the clinical outcome.

Another important issue is the selection of $\mathrm{BCI}$ features that should be utilized for proper feedback. Currently, people are using slow cortical potentials, the delta to alpha ratio, mu, alpha, gamma or beta activity. Furthermore, electrode location is an important consideration for improving classification accuracy as well as the motor function learning. A big question is whether the BCI features should be calculated from brain regions that were affected by the stroke or from healthy regions. Likewise, there are different functional outcome measures, which are selected according to clinical or scientific relevance. Thus, wider acceptance and common measures are of course crucial to interpret results correctly. Questions about more fundamental underlying issues in neuroscience and neuro-plasticity mechanisms also merit discussion. Additionally, in order to compare studies, the patient selection mechanism is important, along with proper communication and generalization of results. Finally, brain stimulation is increasingly being studied, and may play an important role in stroke rehabilitation. 


\section{Future BCI directions}

\section{Causing a sensation: development of a somatosensory afferent interface for BCI users}

Organizer: Lee Miller

Presenters: Kevin Otto, Dustin Tyler, Doug Weber, Sliman Bensmaia, and Philip Sabes

Virtually all BCIs rely exclusively on visual feedback. Yet, lost proprioception causes movements that are poorly coordinated, and require great conscious effort.[140] Likewise, cutaneous anesthesia of the fingers makes manipulating small objects almost impossible.[141] Successful movement BCIs will likely require an afferent interface to convey tactile and proprioceptive feedback in addition to the efferent interface that provides movement.[142,143] Development of a successful somatosensory interface requires addressing the following questions. (1) At what level(s) of the neuroaxis should an interface be made? (2) What stimulus characteristics will maximize efficacy and safety? (3) Is biomimicry a critical design consideration, or does user adaptation make it unnecessary?

Charge-balanced, symmetrical square pulses have long been used to activate the nervous system. Alternative waveforms that have been tested appear to offer little increased efficacy.[144] However, recent results suggest that the dynamics of stimulus train modulation may affect the quality of tactile sensation. In two humans with chronically implanted peripheral nerve cuffs, low-frequency modulation of stimulus intensity transformed the typical tingling sensation into well-localized, graded sensations of pressure that were stable for over a year.[145] The patient reported that the sensations improved prosthesis use and made it feel more like his own limb and less like a tool. Previously, monkeys have learned to use arbitrary intracortical microstimulation (ICMS) trains representing artificial 'textures' to distinguish three virtual objects.[146] Now, well-localized sensations of graded pressure have been produced in monkeys through ICMS in cortical areas $3 \mathrm{~b}$ and 1 using stimulus trains that mimicked natural neuronal somatotopy and dynamics.[147] Future work will tailor the stimulus to convey both temporally precise contact information and a graded sense of contact force.

Both central and peripheral interfaces are also being tested to restore proprioception. One approach in cats has been to record multi-electrode signals under anesthesia from the dorsal root ganglia during passive limb movement.[142] The recordings were 'replayed' through the electrodes, and the stimulus parameters optimized to maximize the similarity between the natural and stimulus-evoked cortical activity.[148] Alternatively, a monkey was trained to report the direction of force perturbations applied to its hand. Electrodes in somatosensory cortical area 2 were characterized by the discharge recorded during movement. In later experiments, the monkey reported a sensation of movement in the corresponding directions when these electrodes were stimulated. A different group used stimulation of eight electrodes in areas 1 and 2 to represent different projections of an error gradient pointing toward a target.[149] They paired the ICMS with visual cues conveying the same information. Although no such error signals are known to exist in S1, after several months of practice two monkeys learned to make accurate movements to targets guided only by the ICMS.

Where possible, an optimal somatosensory interface should likely remain faithful to the principles of sensory representation in S1, while also taking full advantage of natural adaptation. Linking the artificial inputs to well-correlated natural inputs of other modalities may be an effective training strategy. Whether central or peripheral stimulation will ultimately prove more effective remains an open question.

\section{Combining BMI and neural stimulation for restoration of sensory-motor function}

Organizer: Robert Leeb

Presenters: Stanisa Raspopovic, Kai Keng Ang, Joseph E. O'Doherty, and Ricardo Chavarriaga

A brain-machine interface (BMI) typically relies on registering and decoding electric neuronal activity to control external devices. This workshop focused on what may be seen as its counterpart: stimulation of electric neuronal activity, both at the central and peripheral levels, for complementing the use of BMIs by restoring sensory or motor functions, and as a means to effectively close the BMI loop.

Up to now most BMIs have only used visual feedback during control of neuroprosthetic arms.[150] Thus, only movement was restored, not full normal sensorimotor functions of the limbs. However, prosthetic arms are becoming increasingly sophisticated and are already able to provide a sense of touch. Several different ways are explored to establish the new connection between the brain and the prosthetic device for control and sensation.[151] These include using the remaining nerves in the stump, re-routing the nerves to other muscle groups and skins, or directly recording from and stimulating the cortex.

Peripheral neuronal stimulation has been shown to effectively substitute impaired neural pathways to restore motor function in patients with paralysis, providing, for example, sensory feedback for people with upper limb paralysis. Nerve stimulation using transverse intrafascicular multichannel electrodes (TIME) is used in animals, [152] and more recently in humans, to provide information 
about touch and even force pressure from neuroprosthetic devices, enabling a natural grasp.

Intracortical micro-stimulation (ICMS) can be utilized to provide somatosensory feedback, substituting or enhancing sensory capabilities, in what has been termed a bi-directional BCI or brain-machine-brain interface (BMBI). In monkeys, artificial tactile feedback can be created by stimulating the primary somatosensory cortex.[153] Thus, the monkey could perform an active exploration task with a virtual reality arm moved by motor commands derived from neuronal activity recorded from the primary motor cortex, while ICMS feedback occurred whenever the actuator touched a virtual object. This allowed different artificial textures on the virtual objects to be differentiated.[146]

Stimulating the electric neuronal activity in the cortex can be used not only to provide feedback, but also to modulate neuronal excitability. In particular, transcranial direct current stimulation (tDCS) may potentially influence the modulation of electrophysiological patterns exploited by BMI.

Current research addresses the question of whether such stimulation facilitates BMI learning and improves BMI performance.[154] In addition, it is important to assess whether these techniques can selectively enhance the activity of physiologically targeted brain areas, an interesting property for BMI-assisted neurorehabilitation. Clinical studies have shown facilitating effects for upper limb stroke rehabilitation when combining tDCS with motor imagery-based BMI and robotic feedback.[155]

These results suggest that control of motor neuroprostheses by BMIs might benefit from the addition of peripheral or cortical stimulation to either enhance the brain patterns used for control, or to generate artificial sensory feedback from the prosthesis and thereby restore lost sensory-motor function.

\section{Tactile and bone-conduction based BCI paradigms - state of the art, challenges and potential new applications}

Organizers: Tomasz M. Rutkowski and Christoph Guger Presenters: Christoph Kapeller, Mounia Ziat, Moonjeoung Chang, and Hiromu Mori

State-of-the-art stimuli-driven BCI paradigms rely mostly on visual or auditory modalities. Recently tactile (or haptic) modality approaches have been proposed to offer alternative ways to deliver sensory stimulation inputs which could be crucial for patients suffering from weak or lost eyesight or hearing (the so-called 'ear stacking syndrome'). Several techniques have already been developed to connect the BCI to a traditional haptic interface or to utilize those interfaces as stimulation sources. The invited presenters at the workshop presented their recent developments and discussed pros and cons of their approaches.[156-160]

The workshop balanced oral and hands-on interactive presentations, starting with an introduction to basic haptic/tactile concepts, devices, and methods with possible applications to BCI. BCI prototypes described included a tactile-based $\mathrm{BCI}$ involving tactors to deliver P300-generating stimuli,[156] and finger/hand [157,158] and head/bone-conduction $[159,160]$ based tactile BCI prototypes.

Demonstrations provided background on existing haptic devices and illustrated the state of the art and future challenges for tactile BCIs. Audience participation with online tactile BCI prototypes used dry g.SAHARA electrodes for fast EEG set-up. The online demos with tactile BCI-naïve users from the workshop audience did not result in perfect accuracies, but all accuracies were above chance levels. Continued research for this sensory modality is therefore still necessary to identify optimal stimulus generation and evoked response classification.

As illustrated by the modest performance of the online tactile BCI for the workshop's 'tactile modality naïve' users, current tactile BCI paradigms must be modified and improved before tactile BCIs are ready for use by people who are locked-in. However, even in their current form, the tactile BCIs presented may be regarded as a possible alternative for people who are locked-in if they cannot use vision- or auditory-based interfaces due to sensory or other disabilities.

Major lines of study for future research on tactile and bone-conduction BCI paradigms include the possibile bone-conduction sensory effects produced by application of exciters to the head area.[159,160] These concepts have already been applied with healthy users and tests with paralyzed users will follow soon. A tactile BCI with vibrotactile exciters attached to the user's head (scalp bones) is an interesting option for delivery of multimodal stimuli. Somatosensory and auditory stimuli combine via the bone-conduction effect when the stimulation is in an acoustic frequency range. This could provide people with locked-in syndrome or ALS with a potentially fast information transfer rate. $[159,160]$

As a summary of the workshop discussions, a review paper is being developed by the organizers and active workshop participants that focuses on available tactile devices and existing tactile $\mathrm{BCI}$ prototypes. We invite the reader to join us for discussion with other tactile BCI community members through our website (http:// tactile.bci-lab.info), FaceBook page (https://www. facebook.com/tactileBCI) or twitter feed (https:// twitter.com/tactileBCI). 


\section{BCI and detection of consciousness}

Organizer: Christoph Guger

Presenters: Andrea Kübler, Damien Lesenfants, Donatella Mattia, Gernot Müller-Putz, and Srivas Chennu

A recent review showed that 4 of 24 patients (17\%) identified as being in a vegetative state were not only consciously aware but could answer yes or no questions.[161] Other potentially communicative patients with disorders of consciousness might be undetectable through standard clinical testing, but may benefit from BCI. Hence, some users meet all behavioral criteria for vegetative state, but nevertheless have covert awareness. Some research to assess cognitive activity in coma patients relies on fMRI. But fMRI is expensive, bulky, and more time-consuming than EEG assessments. Further, evaluation with fMRI is ineffective in patients with uncontrollable movements and impossible for patients who have metal implants or rely on medical equipment containing metal. EEG-based BCI can provide an effective tool for assessing cognitive state and allowing communication and may be more efficiently used to evaluate patients with transient periods of relative wakefulness.

The workshop featured presentations by coauthors of a new review on BCI and disorders of consciousness. These included presentations on auditory-, tactile-, and motor imagery-based BCI paradigms from the DECODER project $[121,161-163]$ and the usage of auditoryand motor imagery-based BCIs for communication with people who have disorders of consciousness.[164,165] The results of three clinical studies on BCI and disorders of consciousness were presented.[166-170] Finally, the hardware and software components important for a practical assessment and communication system were presented.[161]

A BCI system for this application must manage artifacts and quickly provide sufficient clean data to assess patients and to enable communication. To give medical doctors a useful tool, the BCI system must be trained very quickly and be robust and accurate with patients. The results must be reliable and show with a high degree of certainty whether the patient understands instructions. The system should also allow repeated tests to find fluctuations of consciousness. Real-time feedback of brain activity can be essential by enabling patients to improve BCI performance over time.

Using BCI as a tool in the diagnosis of disorders of consciousness enters a new realm of ethical consideration and medical regulation that is not applicable to technology that is not used for diagnosis. What are the consequences if the BCI system shows that the patient can understand instructions? What if the BCI system shows that the patient cannot? BCI technology to assess, understand, and communicate with patients who have disorders of consciousness may not only change the lives of these patients and their families and caregivers, but may also solve some scientific, clinical, and ethical problems. Thus, results and conclusions of research projects may provide a basis for improved guidelines and new clinical and research procedures.

\section{BCIs for neurodevelopmental disorders}

Organizer: Disha Gupta

Presenters: Scott Makeig, Jaime A. Pineda, Marissa Westerfield, Tzyy-Ping Jung, Leanne Chukoskie, Jonathan Tarbox, and Armin Schnürer

This workshop discussed the need, vision, barriers, and scope of potential BCI interventions in neurodevelopmental disorders such as autism-spectrum disorders (ASD) and attention deficit hyperactivity disorder (ADHD). Neurodevelopmental disorders are estimated to affect 1 in 88 children.[171] ASD encompasses a complex range of neurodevelopmental disorders, characterized by social impairments, communication difficulties, and restricted, repetitive, and stereotyped behavior patterns. Some individuals with ASD are asocial but otherwise high-functioning while others are lowfunctioning and nonspeaking.

ASD intervention research is predominantly done with high-functioning adults or adolescents, perhaps because subjects from the lower extreme of the spectrum may be non-compliant and difficult to manage. However, the younger, lower-functioning groups have the greatest need for early and rapid training, the greatest barriers to normal cognitive development, and potentially would experience the greatest benefit if BCI could improve function or prevent progression of the disorder at an early stage. Currently, the only known effective treatment $[172,173]$ for ASD is intensive one-to-one applied behavior therapy, requiring about $40+$ hours per week for 2-3 years. Further, even this intensive intervention has had mixed success.[172] Limited availability and the high investment of time and money puts even this treatment out of reach for many.

BCI could improve intervention by (a) using direct cognitive features for quantifying responses instead of indirect subjective qualitative behavioral measures; (b) providing rapid real-time feedback and hence increasing the pace of behavioral training by tightening the association between behavior and reward; (c) automating and computerizing the training to be more portable and accessible; and (d) detecting and training some of the difficult-to-measure automatic 'covert' mental states.

BCI research has largely focused on adults with normal cognitive development to improve, restore, enhance or replace disrupted or impaired functional connections. BCI use to supplement or correct atypical cortical development, such as in a neurodevelopmental disorder, may 
appear to be sub-optimal, if not potentially damaging, since neuroscience is only starting to unravel the mysteries of brain development and function. However, considering the severity of cognitive impairment in ASD, experimental interventions to modify attention or basic executive function might be possible with current knowledge, offering hope for improvement to children who have few if any available treatments.

The foundations for BCI intervention in ASD are already in place. Neurofeedback with normative feedback has been used in autism to target improvement in the mirror neuron system [174] or aberrant functional connectivity.[175] EEG features have been identified that can have implications in reshaping behavioral planning.[176,177] Ongoing research projects include combining EEG and body motion capture,[178] vision research in autism,[179] and advanced signal processing methods for extracting useful features from noisy neural datasets.[180]

Challenges to BCI research in low-functioning ASD children include EEG acquisition in non-compliant children and the inevitable artifacts. These challenges may be mitigated through technological solutions such as real-time motion artifact rejection,[181,182] wireless EEG acquisition systems,[183] EEG systems with a dry and easy to apply system [182-185] and EEG hair nets with a high spatial resolution that are easy to drape on the child's head.[186]

While researchers continue to investigate causes and cures for autism, existing BCI expertise could help the current autism/ADHD population who are constantly struggling to manage and cope with the challenges of the disorder.

\section{Passive BCI - using neurophysiological signals that reflect cognitive or affective state}

Organizers: Anne-Marie Brouwer, Thorsten Zander, and Jan van Erp

Presenters: Benjamin Blankertz, Sebastian Grissmann, Manfred Jaschke, and Fabien Lotte

Most current BCIs are intended as alternative output channels to replace lost capabilities such as speech or hand movement. However, brain signals (possibly in combination with other physiological signals) also form an output channel above and beyond the more usual ones, potentially providing continuous, online information about cognitive and affective states without conscious or effortful communication [187-190] (see also the workshop Cognitive processes and brain-machine interfaces above). For example, cognitive workload could be monitored through EEG and skin conductance for adaptive automation. Also, errors could be detected through ERPs and used to correct an erroneous behavioral response. Another potential application was suggested by participants in the Virtual BCI Users'
Forum at this Fifth International BCI Meeting, who included communication of their emotional state among their ideas for future BCI development.

While passive BCIs use neural and physiological responses online, these responses can also be analyzed offline. Examples of this include detecting amygdala responses for neuromarketing and measuring EEG and pupil dilatation as indicators of mental effort for optimizing information systems. This area of applied neurophysiology with offline affective and cognitive-state monitoring already has a long history (see the review of physiological correlates of mental workload by Hancock et al. [191] and an early study on detecting deception by variations in blood pressure [192]). Recent advances in wearable sensor systems, computational power and methods, and online BCIs may enable applications that were previously impossible.

The approximately 50 workshop participants (both scientists and stakeholders) identified challenges for future research in six areas. The most important 'hardware' issue was user-friendliness, involving ease of set-up and user mobility (no wires and miniaturization of equipment). Practical usability was also a focus for the area of 'signal processing', in this case taking the form of methods to avoid calibration and to reduce the number of required channels. Kindermans and Schrauwen [193] presented such a calibration-free P300 speller at the BCI Meeting. For the area of 'Identification of cognitive states', the primary challenge was moving from classical paradigms evoking cognitive processes to real-world situations. Solutions are expected to be found in using context information (also through behavioral data). For 'Identification of affective states', obtaining ground truth was identified as a major challenge for at least some types of applications. 'Applications' to improve individual human-computer interaction were considered the most important or promising. Discussion of 'Ethics' centered on William Casebeer's proposed 'three C's' of bioethics: character (the effect of applied neuroscience on one's own character or virtues), consent (related to privacy issues), and consequence (choose the action that will produce the greatest balance of good over bad consequences). Workshop participants thought consequences to be most important and character to be least important.

The workshop was held in conjunction with organizing a Frontiers in Neuroscience Research Topic 'Using neurophysiological signals that reflect cognitive or affective state'.[194] The first articles are already available. Most articles and a preface are expected to appear in 2014.

\section{Conclusion}

The breadth of the workshop topics and the depth of the research questions presented provide a clear indication of the growing maturity of BCI research. BCIs are 
emerging from a long history of laboratory incubation into the real world of practical use in home environments, with all the challenges, frustrations, and promise of revolutionary benefit for people with the most profound physical limitations that this includes. Some applications are far along the translational arc, but require optimization for real-world success. Other applications are only just being realized or are awaiting feasibility studies. Overall, the workshops of the BCI Meeting Series provided a venue to define the current state-of-the art of $\mathrm{BCI}$ research and a window into the future of $\mathrm{BCI}$ applications.

\section{Acknowledgements}

The authors thank the National Institute on Deafness and other Communication Disorders (NIDCD), Eunice Kennedy Shriver National Institute Of Child Health \& Human Development (NICHD), National Institute Of Biomedical Imaging And Bioengineering (NIBIB), and National Institute Of Neurological Disorders And Stroke (NINDS) in the National Institutes of Health (NIH) for their grant \# R13 DC012744 which supported the BCI Meeting and the travel of many students to the Meeting. The opinions expressed are those of the authors and do not reflect the views of NIDCD, NICHD, NIBIB, NINDS or NIH. We also thank NSF for travel support assisting student attendance at the BCI Meeting.

The workshop organizers thank the members of the Steering Committee for the Fifth International Brain-Computer Interface Meeting: Jane Huggins, Benjamin Blankertz, Febo Cincotti, Janis Daly, Emanuel Donchin, Shangkai Gao, Christoph Guger, Bin He, Leigh Hochberg, Melody Jackson, Andrea Kübler, José del R. Millán, Lee Miller, Koichi Mori, Gernot Müller-Putz, Femke Nijboer, Bijan Pesaran, Nick Ramsey, Gerwin Schalk, Theresa Vaughan, Justin Williams, Catherine Wolf, Jonathan Wolpaw.

Individual Workshop Acknowledgments

The workshop Independent Home Use of BCI was supported by the European ICT Programme Projects FP7-287320 and FP7-288566. This work only reflects the authors' views and funding agencies are not liable for any use that may be made of the information contained herein.

The workshop Augmentative and Alternative Communication for BCI 101 was supported in part by NIH/NIDCD grant 1R01DC009834. The opinions expressed are those of the authors and do not reflect the views of NIDCD or NIH.

The workshop BCI Performance Metrics would like to thank Dr. Cindy Chestek for supporting Dr. Thompson's travel.

The workshop Cognitive Processes and Brain-Machine Interfaces acknowledges the following participants in alphabetical order: A.M. Brouwer (TNO, NL); E. Donchin (U. South Florida, USA); N. Evans (EPFL, CH); R. Leeb (EPFL, CH); J.d.R. Millán (EPFL, CH); T. Mullen (UCSD, USA); S. Rosen (U. Miami); J Sanchez (U. Miami); T. Schultz (KIH, GE); A. Sobolewski (EPFL, CH); A. Soria-Frisch (Starlab, ES);

The workshop Teaching the BCI Skill would like to thank all the participants for the inspiring and insightful discussions that occurred during the workshop.

The workshop Non-invasive BCI-control of Grasp Neuroprosthesis in High Spinal Cord Injured Humans would like to thank T.S. for the demonstration during the workshop. For his participation in our experiments over the last 12 years he received the Olijnyk Award of the International Electrical Stimulation Society (IFESS).
The workshop Current State and Future Challenges in Auditory BCI was partly supported by BrainLinks-BrainTools Cluster of Excellence funded by the German Research Foundation (DFG, grant number EXC 1086).

The workshop Combining BMI and Neural Stimulation for Restoration of Sensory-motor Function would like to acknowledge the particularly active contributions to the discussion by A Soria-Frisch (Starlab, ES); S Bensmaia (U. Chicago, USA); and JdR Millán (EPFL, CH).

The workshop organizers for Tactile and Bone-Conduction Based BCI Paradigms - State of the Art, Challenges and Potential New Applications, T.M. Rutkowski, H. Mori and M. Chang, were supported in part by the Strategic Information and Communications R\&D Promotion Programme no. 121803027 of The Ministry of Internal Affairs and Communication in Japan.

The workshop BCIs for Neurodevelopmental Disorders would like to thank Jeanne Townsend for enthusiastic support of the workshop, Elizabeth Friedrich, Cathrine Dam (EGI net) and Robin Johnson (ABM) for actively participating in the workshop and all the participants for the informative and lively discussions.

The workshop Passive BCI thanks all workshop participants for their active participation!

\section{Notes on Contributors}

Dr. Huggins received a BS in Computer Engineering from Carnegie Mellon in Pittsburgh, Pennsylvania, USA. She received an MS in Bioengineering, an MSE in Computer Engineering, and a $\mathrm{PhD}$ in Biomedical Engineering from the University of Michigan, Ann Arbor. Dr. Huggins is a Research Assistant Professor in the Department of Physical Medicine and Rehabilitation and the Department of Biomedical Engineering at the University of Michigan in Ann Arbor. She leads the University of Michigan Direct Brain Interface project with a goal of making brain-computer interfaces practical for the people who need them. Dr. Huggins served as the chair of the steering committee for the Fifth International BrainComputer Interface Meeting.

Dr. Guger develops invasive and non-invasive real-time biosignal processing systems for brain-computer interface applications. He is active in several international research projects and started g.tec medical engineering $\mathrm{GmbH}$ and Guger Technologies OG to develop this technology.

Dr. Anderson earned a BS degree in Computer Science at the University of Nebraska and $\mathrm{MS}$ and $\mathrm{PhD}$ degrees in Computer Science at the University of Massachusetts, Amherst. He was a Senior Member of Technical Staff at GTE Laboratories in Waltham, Massachusetts, from 1986 through 1990. He joined Colorado State University as an assistant professor of Computer Science in 1991 and is now full professor. Dr. Anderson's research interests are in machine learning algorithms and applications in bio-medical signal analysis and automatic control. 
Dr. Batista received his PhD from Caltech in 1999. He performed postdoctoral research at Stanford. He has been an Assistant Professor in Bioengineering at the University of Pittsburgh since 2007.

Dr. Brouwer received her $\mathrm{PhD}$ from the Erasmus University Rotterdam in the Netherlands in 2002. As a postdoc she studied visuomotor coordination at the Max Planck Institute, Tübingen, Germany, and then at Rochester University, NY, USA. She is currently affiliated with The Netherlands Organization for Applied Scientific Research TNO, Soesterberg, the Netherlands. Her recent and current research focuses on tactile brain-computer interfaces and (possible applications of) physiological correlates of mental state.

Dr. Brunner is a senior postdoctoral researcher at the Institute for Knowledge Discovery, Graz University of Technology, Graz, Austria. In 2011 and 2012, he worked in Scott Makeig's lab at the Swartz Center for Computational Neuroscience, University of California San Diego, La Jolla, CA, USA. He has a total of eight years of experience in the field of BCI research. His research interests include signal processing, machine learning, software development for BCI technology, time series analysis and statistical modeling of biosignals, EEG as a neuroimaging tool (using ICA and source localization), and passive BCIs.

Dr. Chavarriaga is a senior researcher at the Center for Neuroprosthetics of the École Polytechnique Fédérale de Lausanne (EPFL), Switzerland. His research focuses on robust brain-machine interfaces and multimodal humanmachine interaction. Chavarriaga received a $\mathrm{PhD}$ in computational neuroscience from the EPFL.

Dr. Fried-Oken, PhD, is a Professor of Neurology, Biomedical Engineering and Otolaryngology at the Oregon Health \& Science University (OHSU), Director of OHSU Assistive Technology Program, and a clinical speech-language pathologist. As a leading international clinician and researcher in the field of Augmentative and Alternative Communication (AAC), she provides expertise about assistive technology for persons with acquired disabilities who cannot use speech or writing for expression.

Dr. Gunduz, Ph.D. is an Assistant Professor of Biomedical Engineering at the University of Florida, Gainesville. She has a BS in Electrical Engineering from the Middle East Technical University, Ankara, Turkey, and M.S. in Electrical Engineering from North Carolina State University, Raleigh, NC and a Ph.D. in Electrical Engineering from the University of Florida, Gainesville, FL. She completed a post-doctoral fellowship in Neurology at Albany Medical College, Albany, NY.
Dr. Gupta is a Postdoctoral Research Fellow in early brain injury and recovery labs at Burke-Cornell Medical Research Institute, NY. As a Goldsmith Fellow she is pursuing research with humans using neuromodulation for recovery after brain injury. She received her BE (2002) in Electrical Engineering from Punjab University, India and MS (2005) in information communication and system security, from the Royal Institute of Technology, Sweden. She also trained as a Software Systems Engineer (2002-03) at Tata Consultancy Services, India. Her neuroscience training comes from her $\mathrm{PhD}$ research (2004-05) at University of Southampton, UK; Master's research (2004-05) at Fraunhofer Institute for Integrated Circuits, Germany; postdoctoral research (2009-10) at Donders Institute for Brain, Cognition and Behavior, Radboud University, and later (2010-13) at the Wadsworth Brain Computer Interfacing Labs, NY State Dept. of Health and Dept. of Neurology, Albany Medical College, NY.

Prof. Kübler, PhD, Biologist and Psychologist, is Associate Professor at the University of Würzburg and her major research topics within the field of BCI are psychological aspects of BCI control and studies with patients in the field. She is working on using neurofeedback for rehabilitation and therapy.

Dr. Leeb is senior post-doctoral researcher at the Chair in Non-Invasive Brain-Machine Interface, Center for Neuroprosthetics at École Polytechnique Fédérale de Lausanne in Switzerland, where he works on the transfer of BCI technology to patient applications. His research interests include direct brain-computer communication systems, neuro-rehabilitation, biosignal processing, hybrid BCI approaches and virtual reality systems.

Dr. Lotte obtained an MSc (2005), an MEng (2005) and a $\mathrm{PhD}$ degree (2008) in computer science, all from the National Institute of Applied Sciences (INSA), Rennes, France. As a PhD candidate he was part of Inria Rennes Bretagne-Atlantique and member of the OpenViBE project dedicated to brain-computer interfaces and virtual reality. His $\mathrm{PhD}$ thesis received both the $\mathrm{PhD}$ Thesis award 2009 from AFRIF (French Association for Pattern Recognition) and the $\mathrm{PhD}$ Thesis award 2009 accessit (2nd prize) from ASTI (French Association for Information Sciences and Technologies). In 2009 and 2010, he was a research fellow at the Institute for Infocomm Research (I2R) in Singapore, working in the Brain-Computer Interface Laboratory. Since January 2011 he has been a research scientist (with tenure) at Inria Bordeaux Sud-Ouest, France, in team Potioc (http://team.inria.fr/potioc/). His research interests include brain-computer interfaces, pattern recognition, signal processing, and $3 \mathrm{D}$ interaction. 
Dr. Miller received the BA degree in physics from Goshen College, Goshen, IN, in 1980, and the MS degree in biomedical engineering and the $\mathrm{PhD}$ degree in physiology from Northwestern University, Evanston, IL, in 1983 and 1989, respectively. He completed postdoctoral training in the Department of Medical Physics, University of Nijmegen, the Netherlands. He is currently the Edgar C. Stuntz Distinguished Professor of Neuroscience in the Departments of Physiology, Physical Medicine and Rehabilitation, and Biomedical Engineering at Northwestern University. His primary research interests are in the cortical control of muscle activity and limb movement, the representation of limb state by the somatosensory system, and in the development of brain-machine interfaces that attempt to mimic normal physiological systems.

Dr. Müller-Putz is Head of the Institute for Knowledge Discovery, Graz University of Technology, Austria. He is also Head of the Laboratory for Brain-Computer Interfaces (BCI-Lab) at Graz University of Technology. He has gained experience in the field of biosignal analysis, brain-computer interface research, EEG-based neuroprosthesis control, hybrid BCI systems, the human somatosensory system, and assistive technology over the past 12 years. He has organized the main European BrainComputer Interface Conferences over the last 10 years.

Dr. Rutkowski received his MSc in Electronics and $\mathrm{PhD}$ in Telecommunications and Acoustics from Wroclaw University of Technology, Poland, in 1994 and 2002, respectively. He received a postdoctoral training at the Multimedia Laboratory, Kyoto University, and in 2005-2010 he worked as a research scientist at RIKEN Brain Science Institute, Japan. Currently he serves as an assistant professor at the University of Tsukuba and as a visiting scientist at RIKEN Brain Science Institute. Professor Rutkowski's research interests include computational neuroscience, especially brain-computer interfacing technologies, computational modeling of brain processes, neurobiological signal and information processing, multimedia interfaces, and interactive technology design. He is a senior member of IEEE, a member of the Society for Neuroscience, and the AsiaPacific Signal and Information Processing Association (APSIPA).

Dr. Tangermann received his diploma (MSc) in computer science (minor: biology, scholarship of the German National Academic Foundation) in 2000. Michael dived into the field of BCI which grew at the University and Max-Planck Institute (Dept. Empirical Inference) at Tübingen (Germany). There he worked with Wolfgang Rosenstiel, Niels Birbaumer, and Bernhard Schölkopf to introduce machine learning techniques into BCI, for which he earned his $\mathrm{PhD}$ in 2007. By October 2005, he had joined the machine learning research lab of Klaus-Robert Müller and the BBCI group of Benjamin Blankertz in Berlin (Fraunhofer FIRST and Technical University of Berlin). Michael (co-) organized various workshops and BBCI data competitions and co-edited BCI-related special issues. Since 2013 he has been junior research group leader at the University of Freiburg (Germany), where he continues his research on auditory BCIs, patient BCI applications, and $\mathrm{BCI}-$ related machine learning within the cluster of excellence 'BrainLinks-BrainTools'.

Dr. Thompson, Ph.D. received a BS in Electrical Engineering from Kansas State University, Master's degrees in Biomedical Engineering and Electrical Engineering, Systems from the University of Michigan, and a PhD in Biomedical Engineering from the University of Michigan. He completed a postdoc in the Cortical Neural Prosthetics Lab at the University of Michigan. He has accepted a position as an Assistant Professor at Kansas State University.

\section{References}

[1] Wolpaw JR, Birbaumer N, Heetderks WJ, McFarland DJ, Peckham PH, Schalk G, Donchin E, Quatrano LA, Robinson CJ, Vaughan TM. Brain-computer interface technology: A review of the first international meeting. IEEE Trans Rehabil Eng. 2000; 8:164-173.

[2] Vaughan TM, Heetderks WJ, Trejo LJ, Rymer WZ, Weinrich M, Moore MM, Kubler A, Dobkin BH, Birbaumer N, Donchin E, Wolpaw EW, Wolpaw JR. Brain-computer interface technology: A review of the second international meeting. IEEE Trans Neural Syst Rehabil Eng. 2003;11:94-109.

[3] Vaughan TM, Wolpaw JR. The third international meeting on brain-computer interface technology: Making a difference. IEEE Trans Neural Syst Rehabil Eng. 2006; 14:126-127.

[4] Vaughan TM, Wolpaw JR. Special issue containing contributions from the fourth international brain-computer interface meeting. J Neural Eng. 2011;8:020201,2560/8/2/ 020201. Epub 2011 Mar 24.

[5] Frye GE, Hauser CK, Townsend G, Sellers EW. Suppressing flashes of items surrounding targets during calibration of a P300-based brain-computer interface improves performance. J Neural Eng. 2011;8:025024.

[6] Sellers EW. New horizons in brain-computer interface research. Clin Neurophysiol. 2013;124:2-4.

[7] Klobassa DS, Vaughan TM, Brunner P, Schwartz NE, Wolpaw JR, Neuper C, Sellers EW. Toward a highthroughput auditory P300-based brain-computer interface. Clin Neurophysiol. 2009;120:1252-1261.

[8] Mak JN, McFarland DJ, Vaughan TM, McCane LM, Tsui PZ, Zeitlin DJ, Sellers EW, Wolpaw JR. EEG correlates of $\mathrm{P} 300$-based brain-computer interface (BCI) performance in people with amyotrophic lateral sclerosis. J Neural Eng. 2012;9:026014,2560/9/2/026014. Epub 2012 Feb 21.

[9] Kaufmann T, Schulz SM, Grunzinger C, Kubler A Flashing characters with famous faces improves ERP-based brain-computer interface performance. J Neural Eng. 2011;8:056016,2560/8/5/056016. Epub 2011 Sep 20. 
[10] Kaufmann T, Schulz SM, Köblitz A, Renner G, Wessig C, Kübler A. Face stimuli effectively prevent brain-computer interface inefficiency in patients with neurodegenerative disease. Clin Neurophysiol. 2013;124:893-900.

[11] Townsend G, Shanahan J, Ryan DB, Sellers EW. A general P300 brain-computer interface presentation paradigm based on performance guided constraints. Neurosci Lett. 2012;531:63-68.

[12] Kübler A, Mattia D, Rupp R, Tangermann M. Facing the challenge: Bringing brain-computer interfaces to endusers. Artif Intell Med. 2013;59:55-60.

[13] Zickler C, Riccio A, Leotta F, Hillian-Tress S, Halder S, Holz E, Staiger-Salzer P, Hoogerwerf EJ, Desideri L, Mattia D, Kubler A. A brain-computer interface as input channel for a standard assistive technology software. Clin EEG Neurosci. 2011;42:236-244.

[14] Vaughan T, Sellers E, and Wolpaw JR Clinical Evaluation of BCIs In: Wolpaw JR, Winter-Wolpaw E, editors. BrainComputer Interfaces: Principles and Practice. New York: Oxford University Press; 2012. p. 325-336.

[15] Sellers EW, Vaughan TM, Wolpaw JR. A brain-computer interface for long-term independent home use. Amyotrophic Lateral Sclerosis. 2010;11:449-455.

[16] Neumann N, Kubler A. Training locked-in patients: A challenge for the use of brain-computer interfaces. IEEE Trans Neural Syst Rehabil Eng. 2003;11:169-172.

[17] Zickler C, Halder S, Kleih SC, Herbert C, Kübler A. Brain painting: Usability testing according to the user-centered design in end users with severe motor paralysis. Artif Intell Med. 2013;59:99-110.

[18] Kübler A, Holz E, Kaufmann T, Zickler C. (2013). A User Centred Approach for Bringing BCI Controlled Applications to End-Users. In: Reza Fazel-Rezai, editors. Brain-Computer Interface Systems - Recent Progress and Future Prospects. doi: 10.5772/55802. Available from: http://www.intechopen.com/books/brain-computer-interface-systems-recent-progress-and-future-prospects/ a-user-centred-approach-for-bringing-bci-controlled-applications-to-end-users.

[19] Holz EM, Höhne J, Staiger-Sälzer P, Tangermann M, Kübler A. Brain-computer interface controlled gaming: Evaluation of usability by severely motor restricted end-users. Artif Intell Med. 2013;59:111-120.

[20] Riccio A, Leotta F, Bianchi L, Aloise F, Zickler C, Hoogerwerf EJ, Kübler A, Mattia D, Cincotti F. Workload measurement in a communication application operated through a P300-based brain-computer interface. J Neural Eng. 2011;8:025028.

[21] Holz EM, Kaufmann T, Desideri L, Malavasi M, Hoogerwerf E-, Kübler A. User centred design in BCI development. In: Allison BZ, Dunne S, Leeb R, Millán JDR, Nijholt A, editors. Towards Practical Brain-Computer Interfaces. Berlin: Springer; 2013. p. 155-172.

[22] Kaufmann T, Holz EM, Kubler A. Comparison of tactile, auditory, and visual modality for brain-computer interface use: A case study with a patient in the locked-in state. Front Neurosci. 2013;7:129.

[23] Holz, EM, Botrel, L, Kaufmann, T, Kübler, A Independent BCI home-use improves quality of life of a patient in the locked-in state (ALS): A long-term study. Arch Phys Med Rehab. 2014 Special Issue on the 5th International BCI Meeting.

[24] Holz, EM, Botrel, L, Kaufmann, T, Kübler, A Long-Term Independent BCI Home-Use by a Locked-In End-User: An Evaluation Study. In: Proceedings of the 5th Interna- tional Brain-Computer Interface Meeting; 2013; Pacific Grove, California, United States. 2013.

[25] Alles ist möglich Mein Hirn brennt [Internet]. Available from: http://www.startnext.de/mein-hirn-brennt

[26] Kübler A, Holz E, Sellers EW, Vaughan TM. Toward independent home use of BCI: A decision algorithm for selection of potential end-users. Arch Phys Med Rehab. 2014; Special Issue on the Fifth International BCI Meeting.

[27] Beukelman DR, Mirenda P. Augmentative and Alternative Communication. BaltimoreMD: Paul Brookes Publishing; 2013.

[28] Lloyd LL, Fuller DR, Arvidson H. Augmentative and Alternative Communication: A handbook of principles and practices. Needham, MA: Allyn \& Bacon; 1997.

[29] Fager S, Beukelman DR, Fried-Oken M, Jakobs T, Baker J. Access interface strategies. Assist Technol. 2011;24: $25-33$.

[30] Millan JD, Rupp R, Muller-Putz GR, Murray-Smith R, Giugliemma C, Tangermann M, Vidaurre C, Cincotti F, Kubler A, Leeb R, Neuper C, Muller KR, Mattia D. Combining brain-computer interfaces and assistive technologies: State-of-the-art and challenges. Front Neurosci. 2010;4. 10.3389/fnins.2010.00161.

[31] Scherer MJ. Assistive technology: matching device and consumer for successful rehabilition. 1st ed. Washington, DC: American Psychological Association; 2002. $325 \mathrm{p}$.

[32] Light J. Interaction involving individuals using augmentative and alternative communication systems: State of the art and future directions. Augmentative and Alternative Communication. 1988;4:66-82.

[33] White GW, Suchowierska M, Campbell M. Developing and systematically implementing participatory action research. Arch Phys Med Rehabil. 2004;85:3-12.

[34] Huggins JE, Wren PA, Gruis KL. What would braincomputer interface users want? opinions and priorities of potential users with amyotrophic lateral sclerosis. Amyotroph Lateral Scler. 2011 May 2; 12:318-324.

[35] Bieker G, Noethe G, Fried-Oken M. Locked-in and reaching new heights. Speak Up, publication of the United States Society for Augmentative and Alternative Communication. 2011 December; 3-6.

[36] Peters B, Bieker G, Fried-Oken M, Hartman S, Huggins JE, Wolf C, Zeitlin D. BCI users speak up: Feedback on brain-computer interface systems for people with disabilities. Archives of Physical Medicine \& Rehabilitation. 2014; In review

[37] Brunner, C, Andreoni, G, Bianchi, L, Blankertz, B, Breitwieser, C, Kanoh, S, Kothe, CA, Lécuyer, A, Makeig, S, Mellinger, J, Perego, P, Renard, Y, Schalk, G Susila, IP, Venthur, B, Müller-Putz, GR. BCI Software Platforms. In: Allison, BZ, Dunne, S, Leeb, R, Millán, JdR, Nijholt, A, editors. Toward Practical BCIs: Bridging the Gap from Research to Real-World Applications. New York: Springer; 2013; p. 303-331.

[38] Schalk G, McFarland DJ, Hinterberger T, Birbaumer N, Wolpaw JR. BCI2000: A general-purpose brain-computer interface (BCI) system. IEEE Trans Biomed Eng. 2004;51:1034-1043.

[39] Renard Y, Lotte F, Gibert G, Congedo M, Maby E, Delannoy V, Bertrand O, Lécuyer A. OpenViBE: An open-source software platform to design, test, and use brain-computer interfaces in real and virtual environments. Presence. 2010;19:35-53. 
[40] Kothe CA, Makeig S. BCILAB: A platform for braincomputer interface development. J Neural Eng. 2013;10 056014,2560/10/5/056014. Epub 2013 Aug 28.

[41] Oostenveld R, Fries P, Maris E, Schoffelen JM. FieldTrip: Open source software for advanced analysis of MEG, EEG, and invasive electrophysiological data. Comput Intell Neurosci. 2011;2011:156869.

[42] Muller-Putz GR, Breitwieser C, Cincotti F, Leeb R, Schreuder M, Leotta F, Tavella M, Bianchi L, Kreilinger A, Ramsay A, Rohm M, Sagebaum M, Tonin L, Neuper C, Millan Jdel R. Tools for brain-computer interaction: A general concept for a hybrid BCI. Front Neuroinform. 2011;5:30. doi: 10.3389/fninf.2011.00030

[43] Ramoser H, Wolpaw JR, Pfurtscheller G. EEG-based communication: Evaluation of alternative signal prediction methods. Biomed Tech (Berl). 1997;42:226-233.

[44] Bianchi L, Quitadamo LR, Garreffa G, Cardarilli GC, Marciani MG. Performances evaluation and optimization of brain computer interface systems in a copy spelling task. IEEE Trans Neural Syst Rehabil Eng. 2007;15:207216.

[45] Dal Seno B, Matteucci M, Mainardi LT. The utility metric: A novel method to assess the overall performance of discrete Brain-Computer interfaces. Neural Systems and Rehabilitation Engineering. IEEE Transactions. 2010;18: 20-28.

[46] Rehman Laghari KUR, Connelly K. Toward total quality of experience: A QoE model in a communication ecosystem. IEEE. 2012;50:58-65.

[47] Yuan P, Gao X, Allison B, Wang Y, Bin G, Gao S. A study of the existing problems of estimating the information transfer rate in online brain-computer interfaces. J Neural Eng. 2013:026014,2560/10/2/026014. Epub 2013 Feb 28.

[48] Quitadamo LR, Abbafati M, Cardarilli GC, Mattia D, Cincotti F, Babiloni F, Marciani MG, Bianchi L. Evaluation of the performances of different P300 based brain-computer interfaces by means of the efficiency metric. J Neurosci Methods. 2012;203:361-368.

[49] Nijboer F, Clausen J, Allison BZ, Haselager P. The asilomar survey: Stakeholders' opinions on ethical issues related to brain-computer interfacing. Neuroethics. 2013;6:541-578.

[50] Billinger M, Daly I, Kaiser V, Jin J, Allison BZ, Müller-Putz GR, Brunner C. Is it significant? Guidelines for reporting BCI performance. In: Allison BZ, Dunne S, Leeb R, Millan J, Nijholt A, editors. Towards Practical BCIs: Bridging the Gap from Research to Real-World Applications. Berlin: Springer-Verlag; 2013. p. 333-354.

[51] Nijboer F, Clausen J, Allison BZ, Haselager P. Researchers' opinions about ethically sound dissemination of BCI research to the public media. International Journal of Bioelectromagnetism. 2011;13:108-109.

[52] Schalk G, Leuthardt EC. Brain-computer interfaces using electrocorticographic signals. IEEE Rev Biomed Eng. 2011;4:140-154.

[53] Miller KJ, Leuthardt EC, Schalk G, Rao RP, Anderson NR, Moran DW, Miller JW, Ojemann JG. Spectral changes in cortical surface potentials during motor movement. J Neurosci. 2007;27:2424-2432.

[54] Wang W, Collinger JL, Degenhart AD, Tyler-Kabara EC, Schwartz AB, Moran DW, Weber DJ, Wodlinger B, Vinjamuri RK, Ashmore RC, Kelly JW, Boninger ML. An electrocorticographic brain interface in an individual with tetraplegia. PLoS One. 2013;8:e55344.
[55] Kennedy P, Andreasen D, Ehirim P, King B, Kirby T, Mao H, Moore M. Using human extra-cortical local field potentials to control a switch. J Neural Eng. 2004;1: $72-77$.

[56] Yanagisawa T, Hirata M, Saitoh Y, Kishima H, Matsushita K, Goto T, Fukuma R, Yokoi H, Kamitani Y, Yoshimine T. Electrocorticographic control of a prosthetic arm in paralyzed patients. Ann Neurol. 2012;71:353-361.

[57] Chao ZC, Nagasaka Y, Fujii N. Long-term asynchronous decoding of arm motion using electrocorticographic signals in monkeys. Front Neuroeng. 2010;3:3.

[58] Hamer HM, Morris HH, Mascha EJ, Karafa MT, Bingaman WE, Bej MD, Burgess RC, Dinner DS, Foldvary NR, Hahn JF, Kotagal P, Najm I, Wyllie E, Luders HO. Complications of invasive video-EEG monitoring with subdural grid electrodes. Neurology. 2002;58:97-103.

[59] Fountas KN, Smith JR. Subdural electrode-associated complications: A 20-year experience. Stereotact Funct Neurosurg. 2007;85:264-272.

[60] Van Gompel JJ, Worrell GA, Bell ML, Patrick TA, Cascino GD, Raffel C, Marsh WR, Meyer FB. Intracranial electroencephalography with subdural grid electrodes: Techniques, complications, and outcomes. Neurosurgery. 2008;63:498-505-505-506.

[61] Morrell MJ. RNS System in Epilepsy Study Group. Responsive cortical stimulation for the treatment of medically intractable partial epilepsy. Neurology. 2011;77:1295-1304.

[62] Speer, J, Kuhn, R FDA Advisory Panel Recommends Approval of the NeuroPace RNS ${ }^{\circledR}$ System for Medically Refractory Epilepsy [Internet]. 2013. Available from: http://www.neuropace.com/about/news/20130225.html

[63] Torres Valderrama A, Oostenveld R, Vansteensel MJ, Huiskamp GM, Ramsey NF. Gain of the human dura in vivo and its effects on invasive brain signal feature detection. J Neurosci Methods. 2010;187:270-279.

[64] Voytek B, Secundo L, Bidet-Caulet A, Scabini D, Stiver SI, Gean AD, Manley GT, Knight RT. Hemicraniectomy: A new model for human electrophysiology with high spatio-temporal resolution. J Cogn Neurosci. 2010;22: 2491-2502.

[65] Flint RD, Wright ZA, Scheid MR, Slutzky MW. Long term, stable brain machine interface performance using local field potentials and multiunit spikes. J Neural Eng. 2013;10 056005,2560/10/5/056005. Epub 2013 Aug 5.

[66] Rouse AG, Williams JJ, Wheeler JJ, Moran DW. Cortical adaptation to a chronic micro-electrocorticographic brain computer interface. J Neurosci. 2013;33:1326-1330.

[67] Markowitz DA, Wong YT, Gray CM, Pesaran B. Optimizing the decoding of movement goals from local field potentials in macaque cortex. $\mathrm{J}$ Neurosci. 2011;31:18412-18422.

[68] Bouchard KE, Mesgarani N, Johnson K, Chang EF. Functional organization of human sensorimotor cortex for speech articulation. Nature. 2013;495:327-332.

[69] Chang EF, Rieger JW, Johnson K, Berger MS, Barbaro NM, Knight RT. Categorical speech representation in human superior temporal gyrus. Nat Neurosci. 2010;13:1428-1432.

[70] Flinker A, Chang EF, Barbaro NM, Berger MS, Knight RT. Sub-centimeter language organization in the human temporal lobe. Brain Lang. 2011;117:103-109.

[71] Slutzky MW, Jordan LR, Krieg T, Chen M, Mogul DJ, Miller LE. Optimal spacing of surface electrode arrays for 
brain-machine interface applications. J Neural Eng. 2010;7:26004,2560/7/2/026004. Epub 2010 Mar 2.

[72] Treder MS, Schmidt NM, Blankertz B. Gaze-independent brain-computer interfaces based on covert attention and feature attention. J Neural Eng. 2011;8:066003,2560/8/6/ 066003. Epub 2011 Oct 6.

[73] Tonin L, Leeb R, Del R, Millan J. Time-dependent approach for single trial classification of covert visuospatial attention. J Neural Eng. 2012 045011,2560/9/4/ 045011. Epub 2012 Jul 25.

[74] Sajda P, Pohlmeyer E, Jun Wang LC, Parra LC, Christoforou C, Dmochowski J, Hanna B, Bahlmann C, Singh MK, Shih-Fu Chang. In a blink of an eye and a switch of a transistor: Cortically coupled computer vision. Proceedings of the IEEE. 2010;98:462-478.

[75] Kohlmorgen J, Dornhege, G, Braun, ML, Blankertz B, Müller KR, Curio G, Hagemann K, Bruns A Schrauf M, Kincses W Improving human performance in a real operating environment through real-time mental workload detection In: Dornhege G, Millán JdR, Hinterberger T, McFarland D, Müller KR, editors. Toward Brain-Computer Interfacing. Cambridge, MA: MIT press; 2007; p. 409-422

[76] Gopher D, Donchin E. Workload: An Examination of the Concept. In: Boff KR, Kaufman L, Thomas JP, editors. Handbook of Perception and Human Performance, Vol II, Cognitive Processes and Performance. New York: Wiley \& Sons; 1986.

[77] Dennett D Consciousness Explained, Boston, MA: Little Brown \& Company, Boston, MA: 1991.

[78] Sirevaag EJ, Kramer AF, Coles MG, Donchin E. Resource reciprocity: An event-related brain potentials analysis. Acta Psychol (Amst). 1989;70:77-97.

[79] Farwell LA, Donchin E. Talking off the top of your head: Toward a mental prosthesis utilizing event-related brain potentials. Electroencephalogr Clin Neurophysiol. 1988;70:510-523.

[80] Libet B, Gleason CA, Wright EW, Pearl DK. Time of conscious intention to act in relation to onset of cerebral activity (readiness-potential). the unconscious initiation of a freely voluntary act. Brain. 1983;106:623-642.

[81] Lew E, Chavarriaga R, Silvoni S, Jdel R Millan. Detection of self-paced reaching movement intention from EEG signals. Detection of self-paced reaching. Front Neuroeng. 2012;5:13.

[82] Bai O, Rathi V, Lin P, Huang D, Battapady H, Fei DY, Schneider L, Houdayer E, Chen X, Hallett M. Prediction of human voluntary movement before it occurs. Clin Neurophysiol. 2011;122:364-372.

[83] Haggard P. Human volition: Towards a neuroscience of will. Nat Rev Neurosci. 2008;9:934-946.

[84] Muralidharan A, Chae J, Taylor DM. Extracting attempted hand movements from EEGs in people with complete hand paralysis following stroke. Front Neurosci. 2011;5:39.

[85] Khaliliardali Z, Chavarriaga R. Andrei Gheorghe L, Millan Jdel R. Detection of anticipatory brain potentials during car driving. Conf Proc IEEE Eng Med Biol Soc. 2012;2012:3829-3832.

[86] Chavarriaga R. Learning from EEG error-related potentials in noninvasive brain-computer interfaces. IEEE Trans Neural Syst Rehabil Eng. 2010;18:381-388.

[87] Llera A, van Gerven MA, Gómez V, Jensen O, Kappen HJ. On the use of interaction error potentials for adaptive brain computer interfaces. Neural Netw. 2011;24:1120 1127.

[88] Milekovic T, Ball T, Schulze-Bonhage A, Aertsen A, Mehring C. Error-related electrocorticographic activity in humans during continuous movements. J Neural Eng. 2012;9:026007,2560/9/2/026007. Epub 2012 Feb 13.

[89] Iturrate I, Chavarriaga R, Montesano L, Minguez J. Millan Jdel R. Latency correction of error potentials between different experiments reduces calibration time for singletrial classification. Conf Proc IEEE Eng Med Biol Soc. 2012;3288-3291.

[90] Chavarriaga R, Perrin X, Siegwart R. Millan Jdel R. Anticipation- and error-related EEG signals during realistic human-machine interaction: A study on visual and tactile feedback. Conf Proc IEEE Eng Med Biol Soc. 2012;2012:6723-6726.

[91] Ferrez PW. Millan Jd. Simultaneous real-time detection of motor imagery and error-related potentials for improved BCI accuracy. Proceedings 4th Internat BCI. Workshop. 2008;1. 197-202.

[92] Spüler M, Bensch M, Kleih S, Rosenstiel W, Bogdan M, Kübler A. Online use of error-related potentials in healthy users and people with severe motor impairment increases performance of a P300-BCI. Clin Neurophysiol. 2012;123:1328-1337.

[93] Mahmoudi B, Sanchez JC. A symbiotic brain-machine interface through value-based decision making. PLoS One. 2011;6:e14760.

[94] Roset SA, Gonzalez HF, Sanchez JC. Development of an EEG based reinforcement learning brain-computer interface system for rehabilitation. Conf Proc IEEE Eng Med Biol Soc. 2013;2013:1563-1566.

[95] Makeig S, Gramann K, Jung TP, Sejnowski TJ, Poizner H. Linking brain, mind and behavior. Int J Psychophysiol. 2009;73:95-100.

[96] Pfurtscheller G, Allison BZ, Brunner C, Bauernfeind G, Solis-Escalante T, Scherer R, Zander TO, Mueller-Putz G, Neuper C, Birbaumer N. The hybrid BCI. Front Neurosci. 2010:430.

[97] Zhang H, Chavarriaga R. Goel MK, Gheorghe L, Millan Jdel R. Improved recognition of error related potentials through the use of brain connectivity features. Conf Proc IEEE Eng Med Biol Soc. 2012;2012:6740-6743.

[98] Mullen T, Kothe C, Chi YM, Ojeda A, Kerth T, Makeig S, Cauwenberghs G, Jung TP. Real-time modeling and $3 \mathrm{D}$ visualization of source dynamics and connectivity using wearable EEG. Conf Proc IEEE Eng Med Biol Soc. 2013;2013:2184-2187.

[99] Evans N, Blanke O. Shared electrophysiology mechanisms of body ownership and motor imagery. Neuroimage. 2013;64:216-228.

[100] Wolpaw J, Wolpaw EW. Brain-computer interfaces: principles and practice. New York: Oxford University Press; 2012.

[101] Friedrich EV, Neuper C, Scherer R. Whatever works: A systematic user-centered training protocol to optimize brain-computer interfacing individually. PLoS One. 2013;8:e76214.

[102] Faller J, Vidaurre C, Solis-Escalante T, Neuper C, Scherer R. Autocalibration and recurrent adaptation: Towards a plug and play online ERD-BCI. IEEE Trans Neural Syst Rehabil Eng. 2012;20:313-319.

[103] Fruitet J, Carpentier A, Munos R, Clerc M. Automatic motor task selection via a bandit algorithm for a brain- 
controlled button. J Neural Eng. 2013;10:016012,2560/ 10/1/016012. Epub 2013 Jan 21

[104] Lecuyer A, George L, Marchal M. Toward adaptive VR simulators combining visual, haptic, and brain-computer interfaces. IEEE. 2013;33:18-23.

[105] Velasco-Álvarez F, Ron-Angevin R, da Silva-Sauer L, Sancha-Ros S. Audio-Cued Motor Imagery-Based BrainComputer Interface: Navigation through Virtual and Real Environments. Neurocomputing. 2013; 121:89-98.

[106] Scherer R, Proll M, Allison B, Muller-Putz GR. New input modalities for modern game design and virtual embodiment. In: Virtual Reality Short Papers and Posters (VRW) IEEE 2012. 2012;5:163-164.

[107] Grosse-Wentrup M, Scholkopf B. High gamma-power predicts performance in sensorimotor-rhythm brain-computer interfaces. J Neural Eng. 2012;9:046001,2560/9/4/ 046001. Epub 2012 Jun 19.

[108] Wander JD, Blakely T, Miller KJ, Weaver KE, Johnson LA, Olson JD, Fetz EE, Rao RP, Ojemann JG. Distributed cortical adaptation during learning of a brain-computer interface task. Proc Natl Acad Sci USA. 2013;110:10818-10823.

[109] Lotte F, Larrue F, Muhl C. Flaws in current human training protocols for spontaneous brain-computer interfaces: Lessons learned from instructional design. Front Hum Neurosci. 2013;17:568.

[110] Rupp R, Müller-Putz G BCI-Controlled Grasp Neuroprosthesis in High Spinal Cord Injury In: Converging Clinical and Engineering Research in Neurorehabilitation. 2012; p. 1255-9.

[111] Rupp R, Kreilinger A, Rohm M, Kaiser V, Muller-Putz GR. Development of a non-invasive, multifunctional grasp neuroprosthesis and its evaluation in an individual with a high spinal cord injury. Conf Proc IEEE Eng Med Biol Soc. 2012;2012:1835-1838.

[112] Kreilinger A, Kaiser V, Breitwieser C, Williamson J, Neuper C, Muller-Putz GR. Switching between manual control and brain-computer interface using long term and short term quality measures. Front Neurosci. 2011;5: 147.

[113] Muller-Putz GR, Scherer R, Pfurtscheller G, Rupp R. EEG-based neuroprosthesis control: A step towards clinical practice. Neurosci Lett. 2005;382:169-174.

[114] Pfurtscheller G, Muller GR, Pfurtscheller J, Gerner HJ, Rupp R. 'Thought'-control of functional electrical stimulation to restore hand grasp in a patient with tetraplegia. Neurosci Lett. 2003;351:33-36.

[115] Rohm M, Schneiders M, Muller C, Kreilinger A, Kaiser V, Muller-Putz GR, Rupp R. Hybrid brain-computer interfaces and hybrid neuroprostheses for restoration of upper limb functions in individuals with high-level spinal cord injury. Artif Intell Med. 2013;59:133-142.

[116] Muller-Putz GR, Scherer R, Pfurtscheller G, Rupp R. Brain-computer interfaces for control of neuroprostheses: From synchronous to asynchronous mode of operation. Biomed Tech (Berl). 2006;51:57-63.

[117] Kreilinger A, Kaiser V, Rohm M, Leeb R, Rupp R, Müller-Putz G. Neuroprosthesis Control via Non-invasive Hybrid Brain-Computer Interface. 2013.

[118] Rohm M, Rupp R, Schneiders M, Kreilinger A, MüllerPutz GR. Hybrid brain-computer interfaces for control of neuroprosthetic systems for restoration of upper limb functions in high spinal cord injured individuals. Proceedings IFESS. 2013.
[119] Hill NJ, Scholkopf B. An online brain-computer interface based on shifting attention to concurrent streams of auditory stimuli. J Neural Eng. 2012;9:026011,2560/9/2/ 026011. Epub 2012 Feb 15.

[120] Hohne J, Schreuder M, Blankertz B, Tangermann M. A novel 9-class auditory ERP paradigm driving a predictive text entry system. Front Neurosci. 2011;5:99.

[121] Kubler A, Furdea A, Halder S, Hammer EM, Nijboer F, Kotchoubey B. A brain-computer interface controlled auditory event-related potential (p300) spelling system for locked-in patients. Ann N Y Acad Sci. 2009;1157:90-100.

[122] Nijboer F, Furdea A, Gunst I, Mellinger J, McFarland DJ, Birbaumer N, Kubler A. An auditory brain-computer interface (BCI). J Neurosci Methods. 2008;167:43-50.

[123] Riccio A, Mattia D, Simione L, Olivetti M, Cincotti F. Eye-gaze independent EEG-based brain-computer interfaces for communication. $J$ Neural Eng. 2012;9:045001,2560/9/4/045001. Epub 2012 Jul 25.

[124] Schreuder M, Rost T, Tangermann M. Listen, you are writing! speeding up online spelling with a dynamic auditory BCI. Front Neurosci. 2011;5:112

[125] Fallani De Vico, Pichiorri F, Morone G, Molinari M, Babiloni F, Cincotti F, Mattia D. Multiscale topological properties of functional brain networks during motor imagery after stroke. Neuroimage. 2013;83:438-449.

[126] Soekadar SR, Witkowski M, Cossio EG, Birbaumer N, Robinson SE, Cohen LG. In vivo assessment of human brain oscillations during application of transcranial electric currents. Nat Commun. 2013;4:2032.

[127] Ramos-Murguialday A, Broetz D, Rea M, Laer L, Yilmaz O, Brasil FL, Liberati G, Curado MR, Garcia-Cossio E, Vyziotis A, Cho W, Agostini M, Soares E, Soekadar S, Caria A, Cohen LG, Birbaumer N. Brainmachine interface in chronic stroke rehabilitation: A controlled study. Ann Neurol. 2013;74:100-108.

[128] Cho W, Vidaurre C, Hoffmann U, Birbaumer N, Ramos-Murguialday A. Afferent and efferent activity control in the design of brain computer interfaces for motor rehabilitation. Conf Proc IEEE Eng Med Biol Soc. 2011;2011:7310-7315.

[129] Hashimoto Y, Ushiba J, Kimura A, Liu M, Tomita Y. Change in brain activity through virtual reality-based brain-machine communication in a chronic tetraplegic subject with muscular dystrophy. BMC Neurosci. 2010;11:117.

[130] Kasahara T, Terasaki K, Ogawa Y, Ushiba J, Aramaki H, Masakado Y. The correlation between motor impairments and event-related desynchronization during motor imagery in ALS patients. BMC Neurosci. 2012;13:13-66.

[131] Kasashima Y, Fujiwara T, Matsushika Y, Tsuji T, Hase K, Ushiyama J, Ushiba J, Liu M. Modulation of eventrelated desynchronization during motor imagery with transcranial direct current stimulation (tDCS) in patients with chronic hemiparetic stroke. Exp Brain Res. 2012;221:263-268.

[132] Matsumoto J, Fujiwara T, Takahashi O, Liu M, Kimura A, Ushiba J. Modulation of mu rhythm desynchronization during motor imagery by transcranial direct current stimulation. J Neuroeng Rehabil. 2010;27:7-27.

[133] Ono T, Kimura A, Ushiba J. Daily training with realistic visual feedback improves reproducibility of event-related desynchronisation following hand motor imagery. Clin Neurophysiol. 2013;124:1779-1786. 
[134] Ortner R, Irimia DC, Scharinger J, Guger C. A motor imagery based brain-computer interface for stroke rehabilitation. Stud Health Technol Inform. 2012;181:319-323.

[135] Shindo K, Kawashima K, Ushiba J, Ota N, Ito M, Ota T, Kimura A, Liu M. Effects of neurofeedback training with an electroencephalogram-based brain-computer interface for hand paralysis in patients with chronic stroke: A preliminary case series study. J Rehabil Med. 2011;43:951-957.

[136] Takemi M, Masakado Y, Liu M, Ushiba J. Event-related desynchronization reflects downregulation of intracortical inhibition in human primary motor cortex. J Neurophysiol. 2013;110:1158-1166.

[137] Tohyama T, Fujiwara T, Matsumoto J, Honaga K, Ushiba J, Tsuji T, Hase K, Liu M. Modulation of eventrelated desynchronization during motor imagery with transcranial direct current stimulation in a patient with severe hemiparetic stroke: A case report. Keio J Med. 2011;60:114-118.

[138] Yamamoto T, Katayama Y, Ushiba J, Yoshino H, Obuchi T, Kobayashi K, Oshima H, Fukaya C. On-demand control system for deep brain stimulation for treatment of intention tremor. Neuromodulation. 2013;16:230-235.

[139] Hashimoto Y, Ushiba J. EEG-based classification of imaginary left and right foot movements using beta rebound. Clin Neurophysiol. 2013;124:2153-2160.

[140] Sainburg RL, Ghilardi MF, Poizner H, Ghez C. Control of limb dynamics in normal subjects and patients without proprioception. J Neurophysiol. 1995;73:820-835.

[141] Monzee J, Lamarre Y, Smith AM. The effects of digital anesthesia on force control using a precision grip. J Neurophysiol. 2003;89:672-683.

[142] Weber DJ, London BM, Hokanson JA, Ayers CA, Gaunt RA, Torres RR, Zaaimi B, Miller LE. Limb-state information encoded by peripheral and central somatosensory neurons: Implications for an afferent interface. IEEE Trans Neural Syst Rehabil Eng. 2011;19:501-513.

[143] Weber DJ, Friesen R, Miller LE. Interfacing the somatosensory system to restore touch and proprioception: Essential considerations. J Mot Behav. 2012;44: 403-418.

[144] Koivuniemi AS, Otto KJ. The depth, waveform and pulse rate for electrical microstimulation of the auditory cortex. Conf Proc IEEE Eng Med Biol Soc. 2012;2012:2489-2492.

[145] Schiefer MA, Tan DW, Anderson R, Keith M, Schmitt M, Tyler J, Tyler DJ. Restoring sensation in amputees with nerve cuff electrodes. In: BMES Annual Conference. 2013.

[146] O'Doherty JE, Lebedev MA, Ifft PJ, Zhuang KZ, Shokur $\mathrm{S}$, Bleuler H, Nicolelis MA. Active tactile exploration using a brain-machine-brain interface. Nature. 2011;479:228-231.

[147] Tabot GA, Dammann JF, Berg JA, Tenore FV, Boback JL, Vogelstein RJ, Bensmaia SJ. Restoring the sense of touch with a prosthetic hand through a brain interface. Proc Natl Acad Sci USA. 2013;110:18279-18284.

[148] Tomlinson T, Ruiz-torres R, Miller LE. Multi-electrode stimulation in somatosensory area 2 induces a natural sensation of limb movement. In: Annual meeting, Society for Neuroscience; 2013. p. 835.03

[149] Dadarlat MC, O’Doherty JE, Sabes PN. Multisensory integration of vision and intracortical microstimulation for sensory substitution and augmentation. In: Annual meeting, Society for Neuroscience; 2012. p. 292-212
[150] Hochberg LR, Bacher D, Jarosiewicz B, Masse NY, Simeral JD, Vogel J, Haddadin S, Liu J, Cash SS, van der Smagt P, Donoghue JP. Reach and grasp by people with tetraplegia using a neurally controlled robotic arm. Nature. 2012;485:372-375.

[151] Kwok R. Neuroprosthetics: Once more, with feeling. Nature. 2013;497:176-178.

[152] Raspopovic S, Capogrosso M, Badia J, Navarro X, Micera S. Experimental validation of a hybrid computational model for selective stimulation using transverse intrafascicular multichannel electrodes. IEEE Trans Neural Syst Rehabil Eng. 2012;20:395-404.

[153] Berg JA, Dammann JF, 3rd, Tenore FV, Tabot GA, Boback JL, Manfredi LR, Peterson, MLKatyal KD, Johannes MS, Makhlin A, Wilcox R, Franklin RK, Vogelstein RJ, Hatsopoulos NG, Bensmaia SJ. Behavioral demonstration of a somatosensory neuroprosthesis. IEEE Trans Neural Syst Rehabil Eng. 2013;21:500-507.

[154] Chavarriaga, R, Biasiucci, A, Molina, A, RLeeb, Soto León, V, Campolo, M, Oliviero, A, Millán, JdR. tDCS Modulates Motor Imagery-Related BCI Features In: Pons, JL, Torricelli, D, Pajaro, M, editors. Converging Clinical and Engineering Research on Neurorehabilitation. Berlin Heidelberg: Springer, 2013; p. 647-651

[155] Ang KK, Guan C, Phua KS, Wang C, Teh I, Chen CW, Chew E. Transcranial direct current stimulation and EEG-based motor imagery BCI for upper limb stroke rehabilitation. Conf Proc IEEE Eng Med Biol Soc. 2012;2012:4128-4131.

[156] Ortner, R, Rodriguez, J, Guger, C A tactile P300 brain-computer interface, Technical Report 3. Austria: g.tec Medical Engineering GmbH; 2012 December 2012.

[157] Mori, H, Matsumoto, Y, Makino, S, Kryssanov, V, Rutkowski, TM. Vibrotactile Stimulus Frequency Optimization for the Haptic BCI Prototype. In: Proceedings of The 6th International Conference on Soft Computing and Intelligent Systems, and The 13th International Symposium on Advanced Intelligent Systems; 2012; Kobe, Japan. 2012. p. 2150-3

[158] Mori H, Matsumoto Y, Kryssanov V, Cooper E, Ogawa H, Makino S, Struzik Z, Rutkowski TM. Multi-command tactile brain computer interface: A feasibility study. In: Oakley I, Brewster S, editors. Haptic and Audio Interaction Design 2013 (HAID 2013) vol. 7989 of Lecture Notes in Computer Science. Berlin Heidelberg: Springer Verlag; 2013. p. 50-59.

[159] Mori, H, Matsumoto, Y, Struzik, ZR, Mori, K, Makino, S, Mandic, D, Rutkowski, TM. Multi-command tactile and auditory brain computer interface based on head position stimulation. In: Proceedings of the Fifth International Brain-Computer Interface Meeting. 2013; Asilomar Conference Center, Pacific Grove, California, USA. 2013.

[160] Rutkowski, TM, Mori, H, Makino, S, Mori, K Novel spatial tactile and bone-conduction auditory brain computer interface. In: Neuroscience 2013 Abstracts; 2013; San Diego, CA, USA. 2013. p. abstract \#12179, no. 79.03/VV1 in Brain-Machine Interface I.

[161] Guger G, Sorger B, Noirhomme Q, Naci L, Monti MM, Real R, Pokorny C, Veser S, Lugo Z, Quitadamo L, Lesenfants D, Risetti M, Formisano R, Toppi J, Astolfi L, Emmerling T, Heine L, Erlbeck H, Horki P, Kotchoubey B, Bianchi L, Mattia D, Goebel R, Owen AM, Pellas F, Müller-Putz G, Laureys S, Kübler A. Braincomputer interfaces for assessment and communication in disorders of consciousness. In: Naik, Ganesh R, edi- 
tors. Emerging Theory and Practice in Neuroprosthetics. IGIGLOBAL, Hershey, PA: 2014 in press.

[162] Naci L, Monti MM, Cruse D, Kubler A, Sorger B, Goebel R, Kotchoubey B, Owen AM. Brain-computer interfaces for communication with nonresponsive patients. Ann Neurol. 2012;72:312-323.

[163] Kubler A, Kotchoubey B. Brain-computer interfaces in the continuum of consciousness. Curr Opin Neurol. 2007;20:643-649.

[164] Pokorny C, Klobassa D, Pichler G, Erlbeck H, Real R, Kübler A, Lesenfants D, Habbal D, Noirhomme Q, Risetti M, Mattia D, Müller-Putz G. The auditory P300based single-switch brain-computer interface: Paradigm transition from healthy subjects to minimally conscious patients. Artificial intelligence in medicine. 2013; 59:2;81-90.

[165] Müller-Putz G, Pokorny C, Klobassa D, Horki P. A single switch BCI based on passive and imagined movements: Towards restoring communication in minimally conscious patients. International journal of neural systems. 2013;23:2.

[166] Coyle, D, Carroll, A, Stow, J, McCann, A, Ally, A, McElligott, J Enabling Control in the Minimally Conscious State in a Single Session with a Three Channel BCI. In: 1st International Decoder Workshop; 2012. p. 1-4.

[167] Coyle, D, Carroll, Á, Stow, J, McCreadie, K, McElligott, J Visual and Stereo Audio Sensorimotor Rhythm Feedback in the Minimally Conscious State. In: Proceedings of the Fifth International Brain-Computer Interface Meeting; 2013. p. 38-39

[168] Risetti M, Formisano R, Toppi J, Quitadamo LR, Bianchi L, Astolfi L, Mattia D. On ERPs detection in disorders of consciousness rehabilitation. Frontiers in Human Neuroscience. 2013 7:775, 1-10.

[169] Lesenfants, D, Partoune, N, Soddu, A, Lehembre, R, Muller-Putz, G, Laureys, S, Noirhome, Q Design of a novel covert SSVEP-based BCI. In: Proceedings of the 5 th International Brain-Computer Interface Conference. 2011;Graz, Austria. 2011.

[170] Lesenfants D, Habbal D, Lugo Z, Lebeau P, Horki P, Amico E, Pokorny C, Gomez F, Soddu A, Muller-Putz G, Muller-Putz G, Laureys S, Noirhomme Q. Independent SSVEP-based brain-computer interface in locked-in patients. Journal of Neural Engineering. 2014.

[171] Centers for Disease Control. Prevalence of Autism Spectrum Disorders - Autism and Developmental Disabilities Monitoring Network, United States, 2008. 2012; 61: SS03:1-19. Available from http://www.cdc.gov/mmwr/ preview/mmwrhtml/ss6103al.htm

[172] Lovaas OI. Behavioral treatment and normal educational and intellectual functioning in young autistic children. $\mathrm{J}$ Consult Clin Psychol. 1987;55:3-9.

[173] McEachin JJ, Smith T, Lovaas OI. Long-term outcome for children with autism who received early intensive behavioral treatment. Am J Ment Retard. 1993;97:373-391.

[174] Oberman LM, Ramachandran VS, Pineda JA. Modulation of mu suppression in children with autism spectrum disorders in response to familiar or unfamiliar stimuli: The mirror neuron hypothesis. Neuropsychologia. 2008; 46:1558-1565.

[175] Pineda JA, Juavinett A, Datko M. Self-regulation of brain oscillations as a treatment for aberrant brain connections in children with autism. Med Hypotheses. 2012;79:790-798.
[176] Townsend J, Westerfield M, Leaver E, Makeig S, Jung T, Pierce K, Courchesne E. Event-related brain response abnormalities in autism: Evidence for impaired cerebellofrontal spatial attention networks. Brain Res Cogn Brain Res. 2001;11:127-145.

[177] Pineda JA, Brang D, Hecht E, Edwards L, Carey S, Bacon M, Futagaki C, Suk D, Tom J, Birnbaum C, Rork A. Positive behavioral and electrophysiological changes following neurofeedback training in children with autism. Research in Autism Spectrum Disorders. 2008;2:557-581.

[178] Liao, LD, Lin, CT, McDowell, K, Wickenden, AE, Gramann, K, Jung,. TP, Ko, LW, Chang, JY. Biosensor technologies for augmented Brain-Computer interfaces in the next decades. Proceedings of the IEEE. 2012;100:1553-1566.

[179] Rapela J, Lin TY, Westerfield M, Jung TP, Townsend J. Assisting autistic children with wireless EOG technology. Conf Proc IEEE Eng Med Biol Soc. 2012;3504-3506.

[180] Jung TP, Makeig S, Westerfield M, Townsend J, Courchesne E, Sejnowski TJ. Removal of eye activity artifacts from visual event-related potentials in normal and clinical subjects. Clin Neurophysiol. 2000;2012:111:1745-1758.

[181] Gwin JT, Gramann K, Makeig S, Ferris DP. Removal of movement artifact from high-density EEG recorded during walking and running. J Neurophysiol. 2010;103: 3526-3534.

[182] Chi YM, Wang YT, Wang Y, Maier C, Jung TP, Cauwenberghs G. Dry and noncontact EEG sensors for mobile brain-computer interfaces. IEEE Trans Neural Syst Rehabil Eng. 2012;20:228-235.

[183] Matthews R, McDonald NJ, Anumula H, Woodward J, Turner PJ, Steindorf MA, Chang K, Pendleton JM. Novel hybrid bioelectrodes for ambulatory zero-prep EEG measurements using multi-channel wireless EEG system. Foundations of Augmented Cognition Lecture Notes in Computer Science. 2007;4565:137-146.

[184] Liao LD, Wang IJ, Chen SF, Chang JY, Lin CT. Design, fabrication and experimental validation of a novel drycontact sensor for measuring electroencephalography signals without skin preparation. Sensors (Basel). 2011;11:5819-5834.

[185] Guger C, Krausz G, Allison BZ, Edlinger G. Comparison of dry and gel based electrodes for p300 brain-computer interfaces. Front Neurosci. 2012;6:60.

[186] Ryynanen OR, Hyttinen JA, Laarne PH, Malmivuo JA. Effect of electrode density and measurement noise on the spatial resolution of cortical potential distribution. IEEE Trans Biomed Eng. 2004;51:1547-1554.

[187] Coffey, EBJ, Brouwer, A-, Wilschut, ES, van Erp, JBF. Brain-machine interfaces in space: Using spontaneous rather than intentionally generated brain signals. Acta Astronautica. 2010;67:1-11

[188] Zander, T, Kothe, C, Welke, S, Roetting, M. Enhancing Human-Machine systems with secondary input from passive Brain-Computer interfaces. In: Proceedings from the 4th International BCI Workshop and Training Course; 2008; Graz, Austria. 2008

[189] Zander TO, Kothe C. Towards passive brain-computer interfaces: Applying brain-computer interface technology to human-machine systems in general. J Neural Eng. 2011;8:025005,2560/8/2/025005. Epub 2011 Mar 24.

[190] Brouwer, A-, van Erp, JBF, Heylen, D, Jensen, O, Poel, M Effortless Passive BCIs for Healthy Users In: Stephanidis, C, Antona, M, editors. UAHCI/HCII 2013, Part I, 
LNCS 8009. Las Vegas, NV, USA: Springer Berlin Heidelberg; 2013; p. 615-622.

[191] Hancock PA, Meshkati N, Robertson MM. Physiological reflections of mental workload. Aviat Space Environ Med. 1985;56:1110-1114.

[192] Keeler L. A method for detecting deception. American Journal of Police Science. 1930;1:38-51.

[193] Kindermans, P-, Schrauwen, B Dynamic Stopping in a Calibration-less P300 Speller. In: Proceedings of the Fifth International Brain Computer Interface Meeting 2013; 20132013. p. DOI:10.3217/978-3-85125-260-6-75.
[194] Brouwer A-, Zander TO, Van Erp Jan BF Using neurophysiological signals that reflect cognitive or affective state [Internet]. Available from: http://www.frontiersin.org/ neuroprosthetics/researchtopics/using_neurophysiologi cal_signa/1528.

[195] Vuckovic A, Pineda JA, LaMarca K, Gupta D, Guger C. Frontiers in Neuroengineering: Interaction of BCI with the underlying neurological conditions in patients: pros and cons [Internet]. Available from: http://www.frontiersin.org/neuroengineering/researchtopics/ interaction_of_bci_with_the_un/2020 